\title{
PAPR reduction in FBMC using an ACE-based linear programming optimization
}

\author{
Nuan van der Neut ${ }^{*}$, Bodhaswar TJ Maharaj ${ }^{1}$, Frederick de Lange, Gustavo J González², \\ Fernando Gregorio ${ }^{2}$ and Juan Cousseau ${ }^{2}$
}

\begin{abstract}
This paper presents four novel techniques for peak-to-average power ratio (PAPR) reduction in filter bank multicarrier (FBMC) modulation systems. The approach extends on current PAPR reduction active constellation extension (ACE) methods, as used in orthogonal frequency division multiplexing (OFDM), to an FBMC implementation as the main contribution.

The four techniques introduced can be split up into two: linear programming optimization ACE-based techniques and smart gradient-project (SGP) ACE techniques. The linear programming (LP)-based techniques compensate for the symbol overlaps by utilizing a frame-based approach and provide a theoretical upper bound on achievable performance for the overlapping ACE techniques. The overlapping ACE techniques on the other hand can handle symbol by symbol processing. Furthermore, as a result of FBMC properties, the proposed techniques do not require side information transmission. The PAPR performance of the techniques is shown to match, or in some cases improve, on current PAPR techniques for FBMC. Initial analysis of the computational complexity of the SGP techniques indicates that the complexity issues with PAPR reduction in FBMC implementations can be addressed.

The out-of-band interference introduced by the techniques is investigated. As a result, it is shown that the interference can be compensated for, whilst still maintaining decent PAPR performance. Additional results are also provided by means of a study of the PAPR reduction of the proposed techniques at a fixed clipping probability. The bit error rate (BER) degradation is investigated to ensure that the trade-off in terms of BER degradation is not too severe. As illustrated by exhaustive simulations, the SGP ACE-based technique proposed are ideal candidates for practical implementation in systems employing the low-complexity polyphase implementation of FBMC modulators. The methods are shown to offer significant PAPR reduction and increase the feasibility of FBMC as a replacement modulation system for OFDM.
\end{abstract}

Keywords: Cognitive radio; OFDM; PAPR; ACE; SGP; FBMC; Polyphase realization

\section{Introduction}

Frequency spectrum is a finite resource in wireless communications, and therefore, the search for an efficient modulation technique is an important area of research. Due to the ever increasing demand for higher data rate wireless applications, cognitive radio (CR) applications are gaining popularity as a means for spectrum sharing. However, spectrum sharing between opportunistic users and licensed users require that both users utilize a modulation

\footnotetext{
${ }^{*}$ Correspondence: nuanvanderneut@gmail.com

${ }^{1}$ Department of Electrical, Electronic and Computer Engineering, University of Pretoria, cnr. Lynnwood Road and Roper str., 0002 Pretoria, Republic of South Africa

Full list of author information is available at the end of the article
}

method with well-defined (non-interfering) bands. As a result, both kinds of users can coexist and a higher level of total spectral efficiency can be attained.

Orthogonal frequency division multiplexing (OFDM) solutions based on existing mobile access technologies (3GPP Long Term Evolution (LTE) and LTE advanced) cannot solve alone the interference problem due to their high adjacent channel power ratio (ACPR) [1,2]. In fact, OFDM requires additional filtering to meet suitable ACPR, which leads to additional implementation complexity. Furthermore, efficient use of a fragmented spectrum requires a modulation scheme with fast decaying out-of-band (OOB) components, which may not be achievable using OFDM.

\section{Springer}

(c) 2014 van der Neut et al.; licensee Springer. This is an Open Access article distributed under the terms of the Creative Commons Attribution License (http://creativecommons.org/licenses/by/4.0), which permits unrestricted use, distribution, and reproduction in any medium, provided the original work is properly credited. 
Recently, filter bank multicarrier (FBMC) modulation systems have drawn much attention as a viable alternative to OFDM, especially in CR applications [3,4]. FBMC offers increased bandwidth efficiency as well as low OOB interference by employing a bank of well-defined filters with tight spectral characteristics [5]. However, FBMC systems still suffer from high peak-to-average power ratio (PAPR) inherent in multicarrier systems, such as OFDM. A high PAPR greatly degrades the efficiency of high-power amplifiers (HPA), as the HPA must be operated with a large input backoff (IBO) in order to operate in the linear region and avoid clipping [6]. A reduction in the PAPR increases the efficiency that is crucial for mobile transmitters, with limited energy resources.

Several methods have been suggested for OFDM PAPR reduction. These include clipping, decision-aided reconstruction clipping, coding, partial transmission sequence (PTS), selective mapping (SLM), companding transform, tone reservation, active constellation extension (ACE), among others [7].

For FBMC PAPR reduction, a limited number of methods have been proposed. These include overlapping SLM (OSLM), sliding window tone reservation (SWTR) and multi-block joint optimization (MBJO) methods [8-10]. Although these methods do not distort the magnitude of the original constellations, they do require additional side information to be sent reducing the bandwidth efficiency prior to transmission. The SWTR method shows great promise for practical implementation and provides good results. However, the addition of reserved tones results in a bandwidth efficiency loss of $12.5 \%$. The OSLM method suffers from a higher complexity inherent to SLM techniques. The results of OSLM are also negatively impacted when increasing the overlap factor. This is a possible indication that the overlapping nature of FBMC symbols is not addressed fully. A slight improvement on the OSLM method is proposed in [11]. A noticeable drawback is that increasing the number of subcarriers results in a drop in performance. The utilization of $U=4$ codes implies that four modulators are required, or alternatively, each transmitted signal needs to be modulated with four different codes. These results could be improved by utilizing a higher number of codes, but this will result in an increased complexity. The MBJO method provides good results; however, the complexity of utilizing a trellis for the optimization of the ideal PTS sequence would increase computational complexity and may make this method infeasible in practice. Clipping-based PAPR reduction is proposed in [12]. The results appear promising; however, the performance needs to be verified in a realistic scenario. Another method of reducing PAPR is an adaption of the prototype filter. This method has proven to work well in wavelet OFDM [13]. However, in FBMC systems, when considering nearly perfectly orthogonal prototype filters, the different filter shapes have a very small impact on the PAPR of the signals [14].

The ACE method is of particular interest and uses an iterative clipping and filtering process of an up-sampled OFDM signal. In addition to the clipping and filtering, the ACE iteration includes an 'extended constellation' selection that maintains the minimum Euclidean distance of the constellation points of the corresponding OFDM subcarrier symbol [15].

In this work, we propose four novel techniques of PAPR reduction for FBMC systems based on new extended implementations of ACE method as applied in OFDM systems [15]. The proposed methods can be divided into two classes, namely an optimization class and an overlapping ACE class. The optimization-based approaches make use of linear programming (LP) in order to optimize an objective function based on a set of constraints. In addition, the second class of methods proposed, formed by two alternate methods, are based on simplifications of the previous optimization methods as well as an adaptation from smart gradient-project (SGP) ACE extended to FBMC systems. Since ACE is an iterative method and considering complexity constraints, it is of great importance to reduce the required number of iterations for an achievable PAPR. The proposed methods attempt to gain maximum PAPR reduction capabilities in a single iteration in order to lower the overall system complexity.

The outline of the paper is the following. In Section 2 FBMC modulation characteristics and implementation aspects are reviewed. PAPR for OFDM and FBMC are also reviewed in this section. Mainly for comparison purposes, some ACE-based methods for OFDM and FBMC available in the literature are revisited in Section 3. Section 4 introduces the main contributions of this work for FBMC ACEbased PAPR reduction. In this section, two approaches are presented: one based on LP (aiming for the lowest PAPR reduction) and the other based on an extension of the smart gradient-project ACE-based method used for OFDM (aiming for low implementation complexity). In Section 5, the proposed FBMC PAPR reduction schemes are illustrated by computer simulations and are validated by exhaustive comparisons with other PAPR reduction techniques. A brief discussion and complexity analysis are done in Section 5.5 and Section 5.4. Finally, the conclusions are presented in Section 6.

\section{FBMC modulation aspects and PAPR}

FBMC modulated systems consist of a bank of welldefined filters with tight spectral characteristics. These filters are frequency and phase-shifted versions of an original prototype filter designed to comply with Nyquist constraints and provide high-frequency selectivity [16]. The high-frequency selectivity is obtained by increasing the impulse response length of the filter. The prototype 
filter impulse response is designed to be $K$ times longer than the number of subcarriers $N$. Thus, the effective symbol length is increased to a length of $L=K N$. $K$ is also referred to as the overlap factor and determines the number of overlapping complex FBMC symbols per sample. The overlap is required to maintain the same theoretical throughput as that of an OFDM system.

\subsection{FBMC-OQAM}

FBMC requires purely real input symbols. Orthogonal quadrature amplitude modulation (OQAM) is a staggering technique used to transform complex input data symbols into real symbols at twice the sampling rate [16]. OQAM is used in FBMC to ensure that only real symbols are fed into the filter bank. OQAM symbols are analogous to alternating real and imaginary PAM symbols staggered by $N / 2$ samples. The OQAM symbols are then fed into the FBMC modulator, where they are filtered and modulated onto their respective subcarriers. The output of the FBMC modulator at sample $n$ can be written as [17]

$$
\begin{aligned}
s[n]= & \sum_{q=-\infty}^{\infty} \sum_{k=0}^{N-1}\left(\theta_{k} \Re\left\{\tilde{X}_{q}[k]\right\} p[n-q N]\right. \\
& \left.+\theta_{k+1} \Im\left\{\tilde{X}_{q}[k]\right\} p\left[n-q N-\frac{N}{2}\right]\right) e^{j k(n-q N) \frac{2 \pi}{N}}
\end{aligned}
$$

with

$$
\theta_{k}= \begin{cases}1, & \text { if } k \text { is even } \\ j, & \text { if } k \text { is odd }\end{cases}
$$

where $\tilde{X}_{q}[k]$ is the complex input symbol $q$ at subcarriers $k, \Re$ and $\Im$ represent the real and imaginary components respectively and $p[n]$ is the prototype filter.

The OQAM preprocessing stage can also be considered as a source encoding technique. The OQAM encoder is fed with non-return-to-zero (NRZ) data and maps it to alternating real and imaginary symbols that are fed into the FBMC modulator. These symbols are fed at twice the sampling rate to maintain the same data rate as a corresponding OFDM system using a complex data source coding technique [16].

By defining

$$
\begin{aligned}
X_{2 m}[k] & =\Re\left\{\tilde{X}_{m}[k]\right\}, \\
X_{2 m+1}[k] & =\Im\left\{\tilde{X}_{m}[k]\right\}
\end{aligned}
$$

and considering a frame of $M$ pulse amplitude modulation (PAM) symbols, Equation 1 can then be reduced to

$$
s[n]=\sum_{m=0}^{M-1} \sum_{k=0}^{N-1}\left(\theta_{k+m} X_{m}[k] p\left[n-m \frac{N}{2}\right]\right) e^{j 2 \pi k \frac{n}{N}},
$$

where $p[n]$ is the prototype filter. This is analogous to symbols of a PAM system that are transmitted with a time offset of half a symbol duration $T_{0} / 2$, i.e. the effective symbol period is halved thereby retaining the same data rate capabilities as QAM modulated systems with symbol period $T_{0}$. The individual FBMC symbols of length $L$ and fundamental period $T_{0} / 2$ can then be written as

$$
x_{m}[n]=\sum_{k=0}^{N-1} \theta_{k+m} X_{m}[k] p\left[n-m \frac{N}{2}\right] e^{j 2 \pi k \frac{n}{N}} .
$$

Equation 5 allows the output signal of the FBMC modulator $s[n]$ in Equation 4 to be written in terms of its constituent overlapping symbols, as

$$
s[n]=\sum_{m=\left\lfloor\frac{n}{N / 2}\right\rfloor-(2 K-1)}^{\left\lfloor\frac{n}{N / 2}\right\rfloor} x_{m}\left[|n|_{N / 2}+\left(\left\lfloor\frac{n}{N / 2}\right\rfloor-m\right) \frac{N}{2}\right] \text {, }
$$

with $m=[0,1,2, \cdots, M-1],\lfloor x\rfloor$ represents the largest integer not greater than $x$ and $|\cdot|_{N / 2}$ is the module $N / 2$ operation.

By defining

$$
\boldsymbol{x}_{m}=\left[x_{m}[0], \cdots, x_{m}[L-1]\right]^{T} .
$$

Equation 6 can be used to compute the index points of the symbol set $\boldsymbol{x}_{m}, \ldots, \boldsymbol{x}_{m+2 K-1}$, which overlap at each sample $n$ in an FBMC modulated baseband signal $s[n]$. Equation 6 gives an indication of the overlapping nature of the FBMC symbols for a causal system starting at index $n=0$. This representation is important to visualize how to perform PAPR reduction in FBMC by modifying individual symbols in a frame. Figure 1 illustrates the overlapping nature of FBMC, as described by Equation 6, in a frame of $M$ symbols with an overlap factor $K=4$ and $N=4$ subcarriers. It should be clear that the fundamental symbol period $T_{0} / 2$ is equivalent to $N / 2=2$ in the discrete time domain.

\subsection{Polyphase implementation}

A direct form implementation of Equation 1 can be achieved using a transmultiplexer (TMUX) realization. This is implemented using $N$ up-samplers and a filter bank of $N$ branches. However, filtering has to be applied to each up-sampled subcarrier branch at the higher sampling rate, greatly increasing system complexity [18]. A reduction in the complexity of the systems can be obtained with a polyphase realization employing the efficient fast Fourier transform (FFT) [19-21].

The benefit of the polyphase implementation can be attributed to moving the upsampling through the filters and inverse fast Fourier transform (IFFT) processes by using the multirate identity $[19,20]$. This allows the filtering to be applied at a lower sampling rate, greatly reducing 


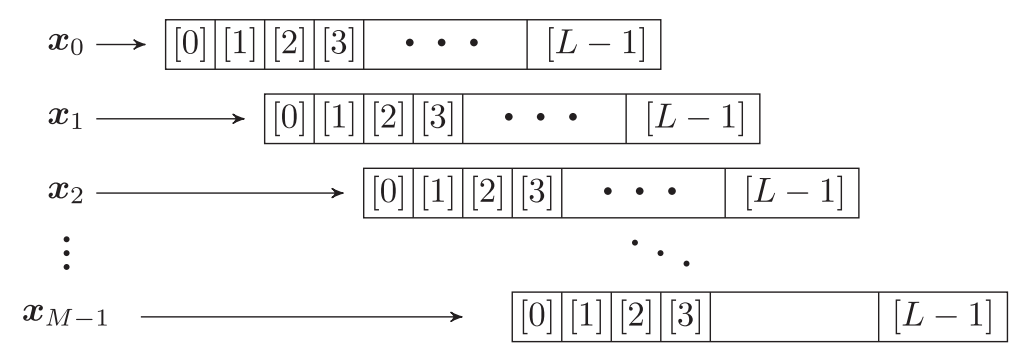

Figure 1 FBMC overlapping nature.

the number of computations required [21]. This is illustrated in Figure 2 with $\tilde{\boldsymbol{X}}\left(z^{N / 2}\right)$ the OQAM processed input symbols, $A_{k}\left(z^{2}\right)$ the low-rate polyphase filters and $Y(z)$ the discrete output.

The delay line in Figure 2 is of length $N$ whereas the up-sampling ratio is $N / 2$ resulting in an effective delay line overlap. This can be seen as compensating for the rate loss due to the OQAM mapping by halving the effective symbol period $T_{0} / 2$. Ignoring the transient build up of the FBMC system, the efficiency of the system can be seen as one complex symbol per sample for a critically sampled implementation. The effective throughput is therefore equivalent to an OFDM system without cyclic prefix $(\mathrm{CP})$.

\subsection{PAPR in multicarrier systems}

The PAPR is an important metric in multicarrier transmission schemes due to their non-constant envelope. Multicarrier systems such as OFDM and FBMC are known to have inherently high PAPRs which can severely degrade the spectral performance of the system or lead to highly inefficient use of HPAs.

\subsubsection{OFDM case}

The PAPR is defined as [22]

$$
\operatorname{PAPR}\left(\mathbf{s}_{m}\right)_{\mathrm{dB}}=10 \log \left(\frac{\max _{0 \leq n \leq N-1}\left|\left\{\mathbf{s}_{m}\right\}_{n}\right|^{2}}{E\left|\mathbf{s}_{m}\right|^{2}}\right)
$$

where $\mathbf{s}_{m}$ is the $m$ th symbol vector of length $N$ and $E\left|\boldsymbol{s}_{m}\right|$ is the expected value of the $m$ th symbol vector.

The cumulative complementary distribution function (CCDF) is an established method of measure for PAPR in multicarrier systems [15]. The CCDF is defined as the probability that the PAPR of the $m$ th modulated symbol $\boldsymbol{s}_{m}$ exceeds a given threshold $\gamma$ and is defined as [15]

$$
\operatorname{CCDF}\left[\operatorname{PAPR}\left(\boldsymbol{s}_{m}\right)\right]=\operatorname{Pr}\left(\operatorname{PAPR}\left(\boldsymbol{s}_{m}\right)>\gamma\right) .
$$

However, it is well established in the literature that in order to accurately estimate the true analogue signal, oversampling of some form needs to be performed [15,23]. In a critically sampled system, Equation 8 is therefore a very optimistic approach to the true PAPR of the symbol.

\subsubsection{FBMC case}

The PAPR for an FBMC symbol is slightly more complicated to analyse. Figure 3 illustrates the overlapping nature of FBMC symbols with $K=4$ [8]. It is clear that after the filtering process is applied, the symbols are extended to a length of $L=K N$. To compensate for OQAM modulation as well as the extended symbol length, the OQAM FBMC symbols are spaced $N / 2$ samples apart, resulting in a symbol period of $T_{0} / 2$ with $2 K$ overlapping symbols at each sample during steady state.

Due to the overlapping nature of FBMC, FBMC symbols cannot be considered in isolation. Instead, we define a frame containing $M$ overlapping FBMC symbols over

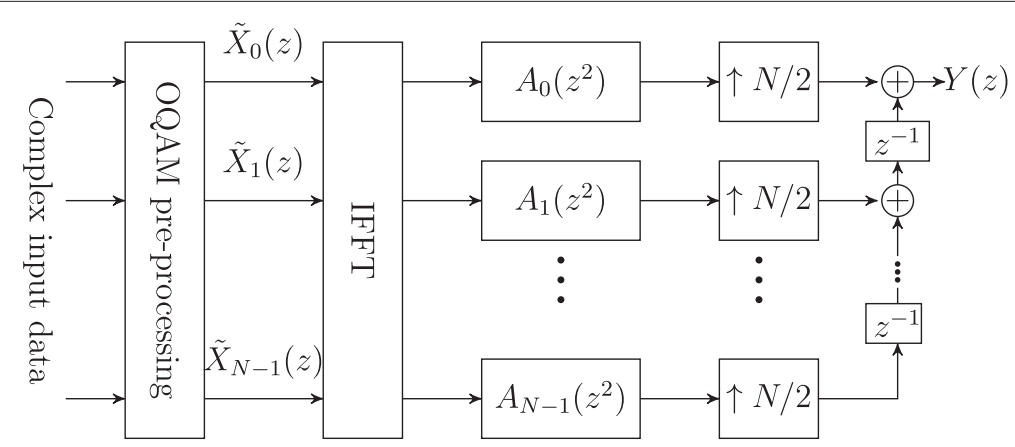

Figure 2 Polyphase implementation of FBMC modulator. 


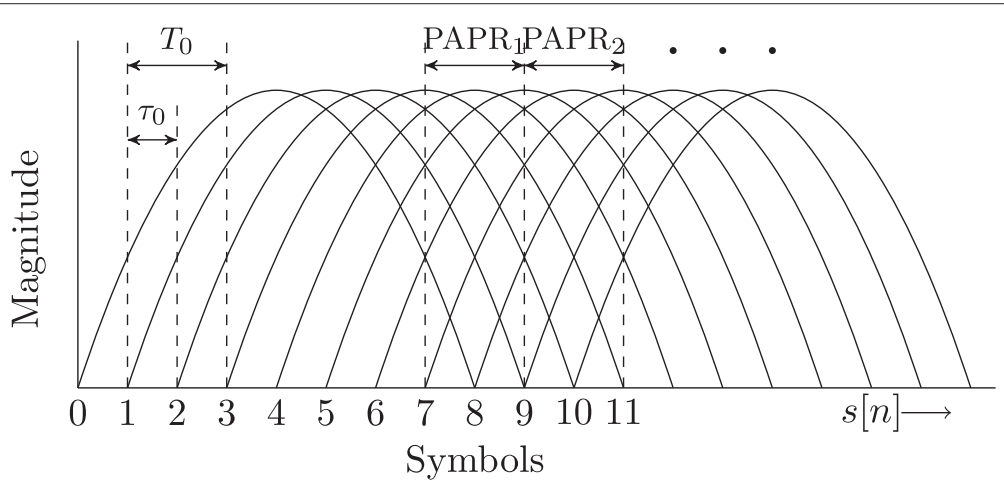

Figure 3 Overlapping nature of FBMC modulation.

which the PAPR can be accurately measured. The output frame is of length $(M-1) N / 2+L$. Two different measurements of PAPR can then be obtained for the FBMC system, namely the frame-based PAPR and the symbolbased PAPR. The PAPR of a frame containing $M$ FBMC symbols can therefore be defined with $s[n]$ the discrete samples, as

$$
\operatorname{PAPR}\left(\mathbf{s}_{l}\right)_{\mathrm{dB}}=10 \log \left(\frac{\max _{0 \leq n \leq(M-1) N / 2+L-1}\left|\left\{\mathbf{s}_{l}\right\}_{n}\right|^{2}}{E\left|\left\{\mathbf{s}_{l}\right\}\right|^{2}}\right)
$$

where $\mathbf{s}_{l}=[s[0], \cdots, s[(M-1) N / 2+L-1]]^{T}$.

For accurate PAPR comparisons to OFDM systems, the FBMC modulated signal $s[n]$ can be broken up into symbols of length $T_{0}$ over which the PAPR can be measured. However, due to the transient build up present in FBMC systems, the PAPR cannot be accurately measured at the start and end of an FBMC frame. Instead, the PAPR should be measured during steady state operation of the system, ideally within a full filter length into the frame and concluding a full filter length before the end of the frame. For simulation purposes, it is necessary to consider a frame length of at least $M=2 K+1$ symbols. This allows for PAPR measure over at least one symbol of length $T_{0}$ in the FBMC frame. Figure 3 illustrates the process of measuring the PAPR over two symbols of length $T_{0}$ each displayed by $\mathrm{PAPR}_{1}$ and $\mathrm{PAPR}_{2}$. Based on that idea, we can define the PAPR of the $v$ th symbol of length $T_{0}$ in a frame as

$$
\operatorname{PAPR}\left(\mathbf{s}_{v}\right)_{\mathrm{dB}}=10 \log \left(\frac{\max _{0 \leq n \leq N-1}\left|\left\{\mathbf{s}_{v}\right\}_{n}\right|^{2}}{E\left|\left\{\mathbf{s}_{v}\right\}\right|^{2}}\right)
$$

where $\mathbf{s}_{v}=[s[L-N / 2+v N], \cdots, s[L-N / 2+(v+1)$ $N-1]]^{T}$.

Equation 11 defines new symbols, $S_{v}$, over which the PAPR can be measured. If the steady state portion of the FBMC frame is split into isolated symbols, the PAPR equation reduces to the same as that of OFDM with symbol period $T_{0}$.

\section{Available ACE-based PAPR reduction techniques for OFDM and FBMC}

\subsection{PAPR reduction using ACE for OFDM}

The ACE algorithm for OFDM PAPR reduction is well documented in the literature [15,24]. It is an efficient method for reducing the PAPR in OFDM without requiring the transmission of side information. The idea behind projection-onto-convex-sets (POCS) of ACE involves clipping the time domain signal and correcting the distorted constellations to only allow extensions that do not decrease the minimum Euclidean distance. This results in PAPR reduction at the expense of a slight increase in average transmit power. The bit error rate (BER) is therefore slightly degraded; however, this is seen as a reasonable trade-off for the PAPR reduction capabilities obtained [15]. The POCS ACE method however requires a relatively large number of iterations to obtain a suitably low PAPR. The SGP method is used to greatly reduce the number of iterations required to obtain a low PAPR. SGP allows to find an optimal scaling factor $\mu$ to scale the clipped portions of the signal. The ACE method for OFDM can be summarized as [15]:

1. Clip the discrete OFDM modulated baseband signal $s[n]$ to an amplitude of $\delta$, with $\phi[n]$ the phase angle of the complex signal $s[n]$ at sampling point $n$, such that

$$
s^{\prime}[n]=\left\{\begin{array}{ll}
\delta e^{j \phi[n]}, & \text { if }|s[n]|>\delta \\
s[n], & \text { if }|s[n]| \leq \delta
\end{array}, \quad 0 \leq n \leq N-1 .\right.
$$

2. Compute the negative of the clipped signal $c[n]$ such that

$c[n]=\left\{\begin{array}{ll}\delta e^{j \phi[n]}-s[n], & \text { if }|s[n]|>\delta \\ 0, & \text { if }|s[n]| \leq \delta\end{array}, \quad 0 \leq n \leq N-1\right.$.

3. Demodulate $c[n]$ to obtain the extension regions $C[k]$ with $k$ the different subcarriers. 
4. Maintain only those real or imaginary components of $C[k]$ which fall within in the allowable extension regions, i.e. those that do not decrease the minimum Euclidean distance and set the rest to zero.

5. For oversampling, set OOB subcarriers to zero, i.e. null all points greater than $N$ [25].

6. Modulate $C[k]$ to obtain the time domain portion of the corrected clipped signal $\hat{c}[n]$.

7. Scale $\hat{c}[n]$ by some constant $\mu$ and add it the original time domain signal $s[n]$ to obtain $\hat{s}[n]$ as

$$
\hat{s}[n]=s[n]+\mu \hat{c}[n], \quad 0 \leq n \leq N-1 .
$$

8. Transmit $\hat{s}[n]$ if it meets the PAPR requirements, otherwise repeat from step (1) replacing $s[n]$ with $\hat{s}[n]$.

\subsection{PAPR reduction using ACE for FBMC}

The POCS ACE method can easily be adapted to be employed in FBMC systems if a frame-based approach is followed. This can be done by following similar steps as POCS ACE for OFDM where $s_{l}[n]$ is a representative of an FBMC modulated baseband frame containing $M$ symbols. POCS can then be summarized as follows:

1. Clip the discrete FBMC modulated baseband frame $s_{l}[n]$ to an amplitude of $\delta$ such that

$s_{l}^{\prime}[n]=\left\{\begin{array}{ll}\delta e^{j \phi[n]}, & \text { if }\left|s_{l}[n]\right|>\delta \\ s_{l}[n], & \text { if }\left|s_{l}[n]\right| \leq \delta\end{array}, \quad 0 \leq n \leq(M-1) N / 2+L-1\right.$.

2. Compute the negative of the clipped frame $c_{l}[n]$ such that

$c_{l}[n]=\left\{\begin{array}{ll}\delta e^{j \phi[n]}-s_{l}[n], & \text { if }\left|s_{l}[n]\right|>\delta \\ 0, & \text { if }\left|s_{l}[n]\right| \leq \delta\end{array}, \quad 0 \leq n \leq(M-1) N / 2+L-1\right.$.

3. Demodulate $c_{l}[n]$ to obtain the extension regions $C_{l, m}[k]$ where $m$ and $k$ represent the frequency domain symbol and subcarrier, respectively, of the FBMC frame 1 .

4. Maintain only those real components of the PAM symbols $C_{l, m}[k]$ which fall within the allowable extension regions and set the rest to zero.

5. For oversampling or digital frequency domain filtering, set OOB (out-of-band) components to zero, i.e. null all subcarriers greater than $N$ [25].

6. Modulate $C_{l, m}[k]$ to obtain the time domain portion of the corrected clipped frame $\hat{c}_{l}[n]$.
7. Scale $\hat{c}_{l}[n]$ by some constant $\mu$ and add it to the original time domain signal $s_{l}[n]$ to obtain $\hat{s}_{l}[n]$

$$
\hat{s}_{l}[n]=s_{l}[n]+\mu \hat{c}_{l}[n], \quad 0 \leq n \leq(M-1) N / 2+L-1 .
$$

8. Transmit $\hat{s}_{l}[n]$ if it meets PAPR requirements, otherwise, repeat from step 1 replacing $s_{l}[n]$ with $\hat{s}_{l}[n]$.

The adapted algorithm for FBMC has to maintain real only constellations as it is required by FBMC-OQAM. This is achieved in step 4 , and the resultant extended constellations can be seen in Figures 4 and 5.

The implementation of SGP ACE for OFDM systems is relatively straight forward due to discrete symbols being obtained, without an overlap. However, the nature of FBMC systems results in multiple overlapping symbols, and therefore, an SGP implementation would require searching for multiple optimal scaling factors requiring additional signal processing. No method currently exists to expand an SGP type implementation of ACE to FBMC. A contribution of this paper is to propose a vectorial extension to the optimal scaling factor $\mu$ for the FBMC frame of $M$ symbols. The main objective of this strategy is to speed up convergence in a similar manner to the SGP method proposed in [15] for OFDM.

\section{Novel PAPR reduction techniques for FBMC}

The proposed PAPR reduction techniques are based on current ACE methods expanded to FBMC applications. Equation 17 allows for scaling of the clipped portion $\hat{c}_{l}[n]$ by a single scaling value across the entire frame, i.e. all symbols in the frame will be scaled by the same value. This strategy does not lead to the best performance since the frame is made up of $M$ overlapping symbols which may be scaled independently.

The main goal is to reduce the number of ACE iterations required to obtain a low PAPR, by finding an extended vectorial scaling factor. This scaling vector allows for independent scaling of each overlapping symbol thereby adding an additional degree of freedom at the cost of additional signal processing.

We can rewrite Equation 17 using a different scaling factor $\mu_{m}$ for each corresponding symbol of the frame as

$$
\begin{aligned}
\hat{\boldsymbol{s}}_{l} & =\boldsymbol{s}_{l}+\hat{\boldsymbol{C}}_{l}^{T} \boldsymbol{\mu}, \\
\boldsymbol{s}_{l} & =[s[0], \cdots, s[(M-1) N / 2+L-1]]^{T},
\end{aligned}
$$

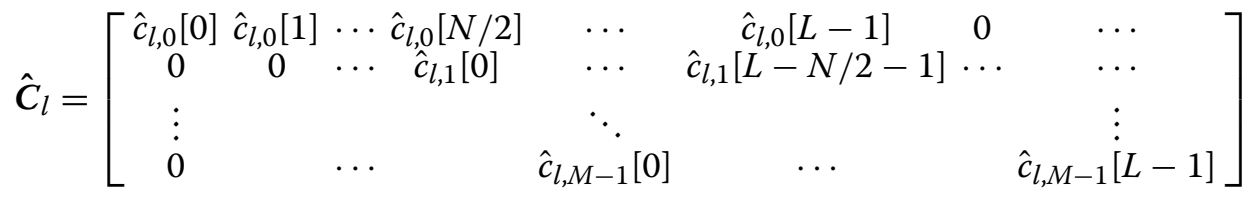




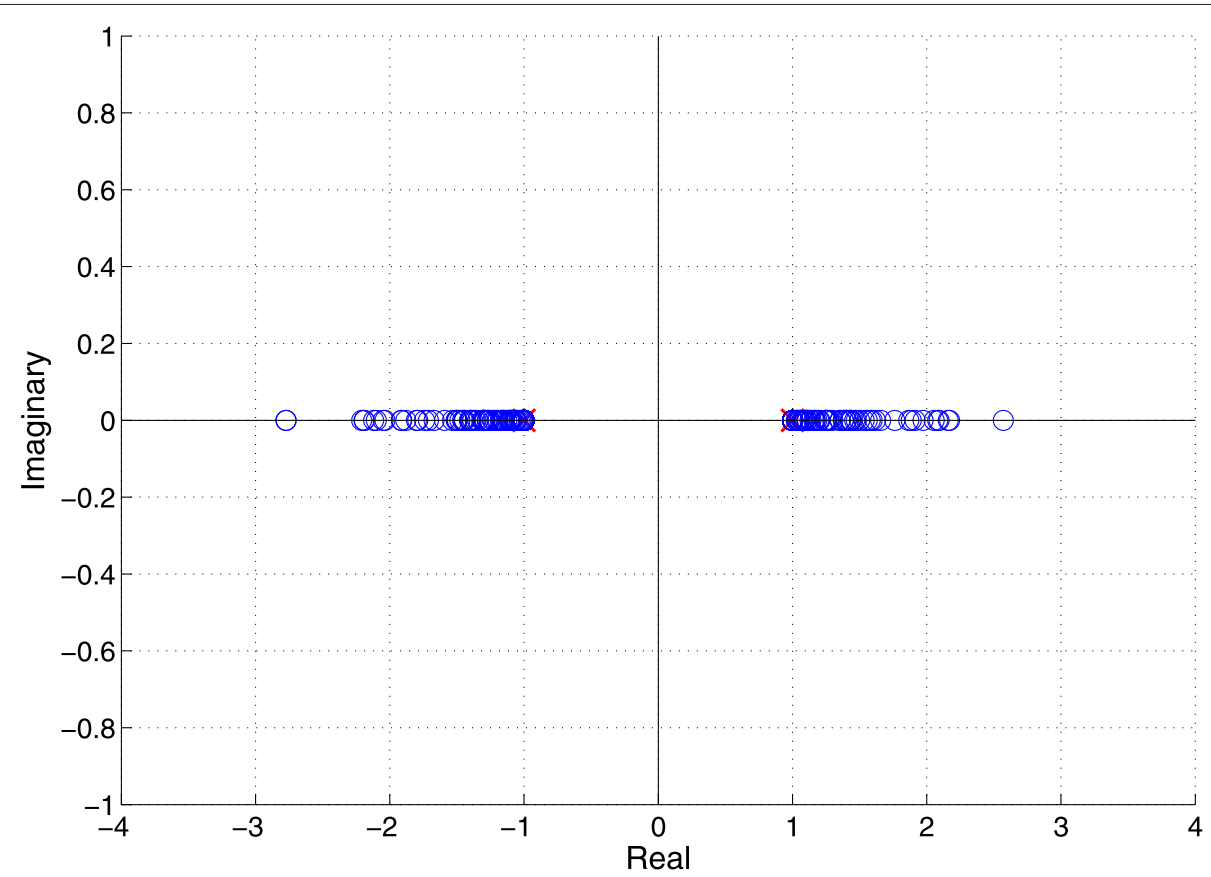

Figure 4 Extended real only constellations for the ACE method adapted to FBMC (QPSK).

$$
\hat{c}_{l, m}[n]=\sum_{k=0}^{N-1} \theta_{k+m} C_{l, m}[k] p\left[n-m \frac{N}{2}\right] e^{j 2 \pi k \frac{n}{N}}
$$

and

$$
\boldsymbol{\mu}=\left[\begin{array}{llll}
\mu_{0} & \mu_{1} & \cdots & \mu_{M-1}
\end{array}\right]^{T} .
$$

From Equation 18, it should be clear that the new baseband FBMC signal, after PAPR reduction, is a function of the individual overlapping clipped symbols, and the rows of matrix $\hat{\boldsymbol{C}}_{l}$ scaled by their relevant scaling factor $\mu_{0} \cdots \mu_{M-1}$ and added to the original FBMC signal $s_{l}$.

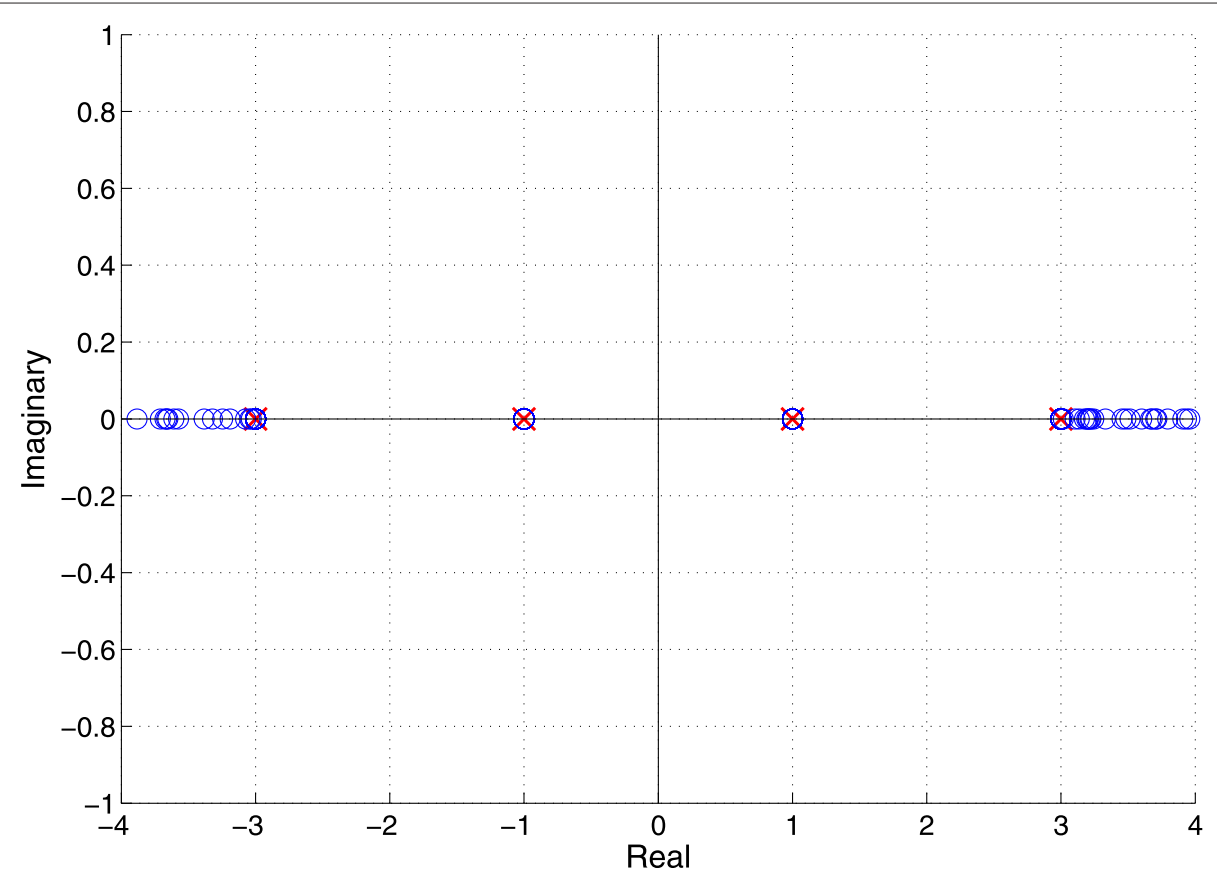

Figure 5 Extended real only constellations for the ACE method adapted to FBMC (16 QAM). 
To obtain the discrete FBMC clipped symbol components of $\hat{\boldsymbol{C}}_{l}$, the individual FBMC symbols must be modulated in a non-contiguous manner. This is required to take into account the effect that each clipped signal has on the original signal looking for the optimal $\mu_{m}$ for $0 \leq m \leq$ $M-1$. This process will reduce much of the efficiency associated with the polyphase implementation.

Figure 6 illustrates the effective overlap of the clipped portions superimposed over the original baseband signal. Each sample of $\hat{\boldsymbol{s}}_{l}$ can then be seen as a function of the original baseband signal $s_{l}$ as well as $2 K$ samples of the overlapping clipped symbols $\hat{c}_{l, m}[n]$. Convergence time can therefore be decreased by optimizing the $\mu_{m}$ to increase the effect that the clipped signals have on reducing the peaks in the original baseband signal. However, the $\mu_{m}$ must be scaled carefully in order to avoid peak regrowth.

\subsection{LP-based optimization}

The authors propose two methods, based on an LP optimization formulation, for finding the optimal scaling factors $\mu_{m}$. In order to provide the largest amount of PAPR reduction, the optimization aims to maximize the $\mu_{m}$ scaling factors. The scaling factor is applied to the negative of the clipped portion of the signal, and therefore, this is equivalent to minimizing the peaks of the signal. Constraints, however, have to be placed on the $\mu_{m}$ values in order to prevent peak regrowth. These constraints provide tight bounds on the LP formulation which guarantee convergence to a lower or equal PAPR per iteration.

The second term of the right hand side of Equation 18 can be written as

$$
\hat{\boldsymbol{C}}_{l}^{T} \boldsymbol{\mu}=\sum_{m=\left\lfloor\frac{n}{N / 2}\right\rfloor-(2 K-1)}^{\left\lfloor\frac{n}{N / 2}\right\rfloor} \mu_{m} \hat{c}_{l, m}\left[|n|_{N / 2}+\left(\left\lfloor\frac{n}{N / 2}\right\rfloor-m\right) \frac{N}{2}\right],
$$

with

$n=[0, \cdots,(M-1) N / 2+L-1], \quad m=[0,1,2, \cdots, M-1]$.

It should be noted that, in order to keep the symbol causal and limit the frame length, the summation limits in Equation 23 are limited by the constraints for $m$ in Equation 24. As an example, for a critically sampled FBMC system with $N=16$ subcarriers and a frame length of $M=25$,

$$
\begin{aligned}
\left\{\hat{\boldsymbol{C}}_{l}^{T} \boldsymbol{\mu}\right\}_{150}= & \mu_{11} \hat{c}_{l, 11}[62]+\mu_{12} \hat{c}_{l, 12}[54]+\mu_{13} \hat{c}_{l, 13}[46] \\
& +\mu_{14} \hat{c}_{l, 14}[38]+\mu_{15} \hat{c}_{l, 15}[30]+\mu_{16} \hat{c}_{l, 16}[22] \\
& +\mu_{17} \hat{c}_{l, 17}[14]+\mu_{18} \hat{c}_{l, 18}[6]
\end{aligned}
$$

with $\{\cdot\}_{n}$ representative of the $n$th component of the vector.

\subsubsection{LP formulation 1}

In order to prevent peak regrowth, the constraints for each sampling point are written in terms of the $\mu_{m}$ scaling factors. The constraints for sample $n$ of frame $l$ can be written, with $n_{\text {peak }}$ the position of the peak as follows:

$$
\begin{aligned}
\left\|s_{l}[n]+\left\{\hat{\boldsymbol{C}}_{l}^{T} \boldsymbol{\mu}\right\}_{n}\right\| & \leq\left\|s_{l}\left[n_{\text {peak }}\right]+\left\{\hat{\boldsymbol{C}}_{l}^{T} \boldsymbol{\mu}\right\}_{n_{\text {peak }}}\right\| \\
n & =[0,1,2, \cdots,(M-1) N / 2+L-1],
\end{aligned}
$$

with

$$
\begin{aligned}
\left\{\hat{\boldsymbol{C}}_{l}^{T} \boldsymbol{\mu}\right\}_{n_{\text {peak }}}= & \sum_{m=\left\lfloor\frac{n_{\text {peak }}}{N / 2}\right\rfloor-(2 K-1)}^{\left\lfloor\frac{n_{\text {peak }}}{N / 2}\right\rfloor} \mu_{m} \hat{\boldsymbol{c}}_{l, m}\left[\left|n_{\text {peak }}\right|_{N / 2}\right. \\
& \left.+\left(\left\lfloor\frac{n_{\text {peak }}}{N / 2}\right\rfloor-m\right) \frac{N}{2}\right] .
\end{aligned}
$$

Equation 26 implies that at each sample $n$, the magnitude of the baseband transmit signal $s_{l}[n]$ combined with the scaled $2 K$ clipped symbols overlapping at $n$

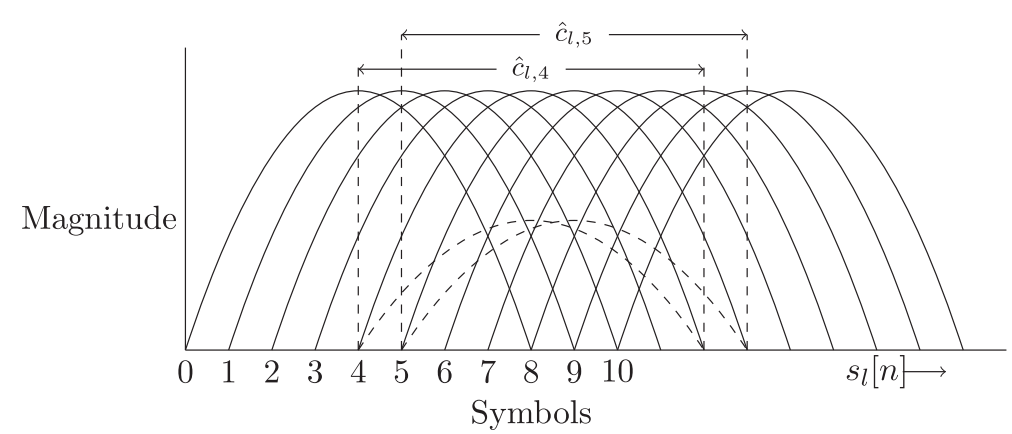

Figure 6 Clipped signals superimposed over original FBMC modulated signal. 
$\left(\left\{\hat{\boldsymbol{C}}_{l}^{T} \boldsymbol{\mu}\right\}_{n}\right)$ cannot exceed the value of the peak of the frame combined with the scaled clipped symbols overlapping at the peaks position (the right hand side of Equation 26). As a consequence, the sample cannot be increased to a magnitude larger than that of the peak after it has been reduced. These constraints limit possible peak regrowth and place a tight upper bound on the problem formulation.

In order to reduce the complexity of an LP-based method, it is desirable to have purely real samples. The output of the FBMC modulator is however a complex baseband signal $s[n]$. For this reason, utilizing a similar approach to that of [15], the magnitude of the sample $\|s[n]\|$ and the projections of the clipped symbols onto the original signal $\bar{c}_{m}[n]$ are used in order to obtain real values for the optimization problem. These projections provide good approximations of the magnitude of the clipped symbols in the same direction as the original signal and can be calculated as [15]

$$
\bar{c}_{l, m}[n]=\frac{\Re\left\{\hat{c}_{l, m}[n] \cdot s_{l}[n]\right\}}{\left\|s_{l}[n]\right\|} .
$$

Equation 28 can be used to estimate Equation 26 by replacing $\left\{\hat{\boldsymbol{C}}_{l}^{T} \boldsymbol{\mu}\right\}_{n}$ with $\left\{\overline{\boldsymbol{C}}_{l}^{T} \boldsymbol{\mu}\right\}_{n}$. The new equation is therefore purely real in nature as it is a representative of magnitude only and can be written as

$$
\begin{aligned}
& \left\|s_{l}[n]\right\|+\left\{\overline{\boldsymbol{C}}_{l}^{T} \boldsymbol{\mu}\right\}_{n} \leq\left\|s_{l}\left[n_{\text {peak }}\right]\right\|+\left\{\overline{\boldsymbol{C}}_{l}^{T} \boldsymbol{\mu}\right\}_{n_{\text {peak }}} \\
& \text { for } n=0, \cdots,(M-1) N / 2+L-1
\end{aligned}
$$

with $\overline{\boldsymbol{C}}_{l}$ a matrix of the same form as Equation 20 with $\hat{c}$ replaced by $\bar{c}$. After moving the constants to the right hand side and the variables to the left, Equation 29 can be written as

$$
\left\{\overline{\boldsymbol{C}}_{l}^{T} \boldsymbol{\mu}\right\}_{n}-\left\{\overline{\boldsymbol{C}}_{l}^{T} \boldsymbol{\mu}\right\}_{n_{\text {peak }}} \leq\left\|s_{l}\left[n_{\text {peak }}\right]\right\|-\left\|s_{l}[n]\right\| .
$$

As mentioned, Equation 29 provides a tight upper bound for the LP problem. To guarantee convergence, a lower bound is also required in the optimization formulation. A good lower bound can be formulated by ensuring that the negative of the absolute value of the scaled samples at each sampling point is greater than the negative of the absolute value of the scaled peak. This can be formulated by adding an extra set of $(M-1) N / 2+L$ constraints,

$$
\begin{aligned}
& -\left\|s_{l}[n]\right\|+\left\{\overline{\boldsymbol{C}}_{l}^{T} \boldsymbol{\mu}\right\}_{n} \geq-\left\|s_{l}\left[n_{\text {peak }}\right]\right\|+\left\{\overline{\boldsymbol{C}}_{l}^{T} \boldsymbol{\mu}\right\}_{n_{\text {peak }}} \\
& \text { for } n=0, \cdots,(M-1) N / 2+L-1,
\end{aligned}
$$

As in Equation 30, Equation 31 can be written with all constants on the right and variables on the left resulting in the final optimization formulation being written as

$$
\max \mathrm{z}=\mu_{0}+\mu_{1}+\cdots+\mu_{M-1}
$$

s.t.

$$
\begin{aligned}
& \left\{\overline{\boldsymbol{C}}_{l}^{T} \boldsymbol{\mu}\right\}_{n}-\left\{\overline{\boldsymbol{C}}_{l}^{T} \boldsymbol{\mu}\right\}_{n_{\text {peak }}} \leq\left\|s_{l}\left[n_{\text {peak }}\right]\right\|-\left\|s_{l}[n]\right\|, \\
& \text { for } n=0, \cdots,(M-1) N / 2+L-1, \\
& \left\{\overline{\boldsymbol{C}}_{l}^{T} \boldsymbol{\mu}\right\}_{n}-\left\{\overline{\boldsymbol{C}}_{l}^{T} \boldsymbol{\mu}\right\}_{n_{\text {peak }}} \geq-\left\|s_{l}\left[n_{\text {peak }}\right]\right\|+s_{l}[n] \| \\
& \text { for } n=0, \cdots,(M-1) N / 2+L-1, \\
& \mu_{m} \geq 0, \quad \text { for } m=0, \cdots, M-1 .
\end{aligned}
$$

This algorithm is an approximation due to the samples being complex in nature and the projections being an approximation of the 'growth' of the clipped signals in the direction of the original baseband signal.

\subsubsection{LP formulation 2}

The aim of LP2 is to minimize the PAPR of sections of the frame instead of over the entire frame as in LP1. This approach therefore handles smaller sections and can achieve superior PAPR performance with a lower average transmit power increase. This is done by changing the optimization formulation. Instead of attempting to reduce a single peak in the frame, the goal is to reduce $M$ peaks in overlapping sections of length $L$. The objective function is also scaled based on the magnitude of the peaks. This allows for higher priority on the larger peaks. In order to do this, we define a new peak position vector $\boldsymbol{n}_{\text {peak }}$ which can be defined as

$$
\boldsymbol{n}_{\text {peak }}=\left[\begin{array}{llll}
n_{\text {peak } 0} & n_{\text {peak } 1} & \cdots & n_{\text {peak } M-1}
\end{array}\right]^{T},
$$

with $n_{\text {peak } 0}$ the position of the peak on the interval $0 \leq$ $n \leq L-1, n_{\text {peak } 1}$ the position of the peak on the interval $N / 2 \leq n \leq L+N / 2-1$ and so on. These peaks set the limits for the intervals of length $N / 2$.

The new optimization formulation can be set up as follows:

$\max \mathrm{z}=\mu_{0}+\mu_{1}+\cdots+\mu_{M-1}$

subject to the following constraints

$$
\left\{\overline{\boldsymbol{C}}_{l}^{T} \boldsymbol{\mu}\right\}_{n}-\left\{\overline{\boldsymbol{C}}_{l}^{T} \boldsymbol{\mu}\right\}_{n_{\text {peak } 0}} \leq\left\|s_{l}\left[n_{\text {peak } 0}\right]\right\|-\left\|s_{l}[n]\right\|,
$$

for $n=0,1, \cdots, L-1$,

$$
\left\{\overline{\boldsymbol{C}}_{l}^{T} \boldsymbol{\mu}\right\}_{n}-\left\{\overline{\boldsymbol{C}}_{l}^{T} \boldsymbol{\mu}\right\}_{n_{\text {peak } 0}} \geq-\left\|s_{l}\left[n_{\text {peak } 0}\right]\right\|+\left\|s_{l}[n]\right\|,
$$

for $n=0,1, \cdots, L-1$, 


$$
\begin{gathered}
\left\{\overline{\boldsymbol{C}}_{l}^{T} \boldsymbol{\mu}\right\}_{n}-\left\{\overline{\boldsymbol{C}}_{l}^{T} \boldsymbol{\mu}\right\}_{n_{\text {peak } 1}} \leq\left\|s_{l}\left[n_{\text {peak } 1}\right]\right\|-\left\|s_{l}[n]\right\|, \\
\text { for } \frac{N}{2} \leq n \leq L+\frac{N}{2}-1, \\
\left\{\overline{\boldsymbol{C}}_{l}^{T} \boldsymbol{\mu}\right\}_{n}-\left\{\overline{\boldsymbol{C}}_{l}^{T} \boldsymbol{\mu}\right\}_{n_{\text {peak } 1}} \geq-\left\|s_{l}\left[n_{\text {peak } 1}\right]\right\|+\left\|s_{l}[n]\right\|, \\
\text { for } \frac{N}{2} \leq n \leq L+\frac{N}{2}-1, \\
\quad \vdots \\
\left\{\overline{\boldsymbol{C}}_{l}^{T} \boldsymbol{\mu}\right\}_{n}-\left\{\overline{\boldsymbol{C}}_{l}^{T} \boldsymbol{\mu}\right\}_{n_{\text {peak } M-1}} \leq\left\|s_{l}\left[n_{\text {peak } M-1}\right]\right\|-\left\|s_{l}[n]\right\|, \\
\text { for } \frac{N}{2}(M-1) \leq n \leq \frac{N}{2}(M-1)+L-1, \\
\left\{\overline{\boldsymbol{C}}_{l}^{T} \boldsymbol{\mu}\right\}_{n}-\left\{\overline{\boldsymbol{C}}_{l}^{T} \boldsymbol{\mu}\right\}_{n_{\text {peak } M-1}} \geq-\left\|s_{l}\left[n_{\text {peak } M-1}\right]\right\|+\left\|s_{l}[n]\right\|, \\
\text { for } \frac{N}{2}(M-1) \leq n \leq \frac{N}{2}(M-1)+L-1,
\end{gathered}
$$

$$
\mu_{m} \geq 0, \quad \text { for } m=0, \cdots, M-1 \text {. }
$$

The objective function of Equation 37 can also be scaled by the relevant peak magnitudes to which the symbols contribute. This adds priority to larger peaks. This can be done by noting the projections at the peak positions of the clipped symbols. The larger peaks will result in larger projections, and therefore, these projections can be used as a scaling factors in the objective function of Equation 37 in order to speed up convergence time to a lower PAPR.

\subsection{SGP extension}

Two additional methods are proposed based on a more direct extension of the SGP ACE method to FBMC. These methods do not require the use of a linear programming methodology, which can have high computational complexity depending on the problem. Once again, both methods focus on finding an optimal scaling factor $\mu$ or scaling factor vector $\mu$.

\subsubsection{SGP single scaling}

This method follows closely to that of the SGP proposed in [15] for OFDM systems. The focus is on finding a single scaling factor $\mu$ by which we can scale the entire clipped frame $\hat{c}_{l}[n]$ given by Equation 17 . This approach can be seen as suboptimal as the component clipped signals can be scaled independently for greater effect.

The proposed single scaling method does not differentiate between the individual symbols in the FBMC frame.

The implementation of the single scaling SGP method is relatively straight forward and can be seen as a generalization of the OFDM method in [15] to an FBMC implementation. Once the clipped portion of the signal across the entire frame is obtained, it can be superimposed over the original signal to obtain the projections. This is illustrated in Figure 7.

The projections are obtained in a manner similar to Equation 28 but with a single vector spanning across the entire frame. In order to obtain the best suited scaling factor $\mu$ for the entire clipped frame $\hat{c}_{l}[n]$, we observe only the projections in the steady state part of the frame, as illustrated by the solid line of the arc in Figure 7. The samples in the transient state are often small in magnitude as they do not contain $2 K$ overlapping symbols and as can be seen in Figure 7 and are scaled by a small magnitude of the corresponding filter response. The signal behaviour in the transient is therefore erratic and not accurate for use in calculating the $\mu$ value. The steady state section begins approximately a full filter length into the frame and ceases a filter length from the end of the frame.

The single scaling SGP method for FBMC requires some alterations of the method in [15] and can be summarized as follows:

1. Find the peak of the frame, $P_{l}$, inside the steady state region (the peak should almost always exist in the

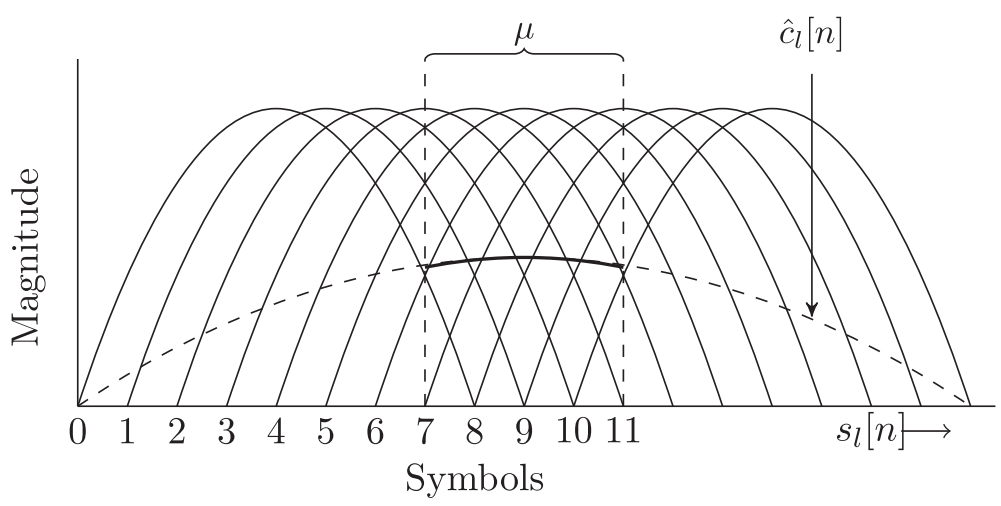

Figure 7 Clipped signals superimposed over original FBMC modulated signal for $M=11$. 
steady state region of the transmit data block) as well as the peak position $n_{\text {peak }}$

2. Calculate the projections $\bar{c}_{l}[n]$ of the clipped frame $\hat{c}_{l}[n]$ only along the viable interval by performing the dot product

$$
\bar{c}_{l}[n]=\frac{\Re\left\{\hat{c}_{l}[n] \cdot s_{l}[n]\right\}}{\left|s_{l}[n]\right|}
$$

for

$$
L-N / 2 \leq n \leq(M-1) N / 2 .
$$

3. Consider only the values of $\bar{c}_{l}[n]$ that are positive and therefore result in magnitude growth.

4. Compute the scaling factor for each of these projections

$$
\mu[n]=\frac{P_{l}-\left|s_{l}[n]\right|}{\bar{c}_{l}[n]-\bar{c}_{l}\left[n_{\text {peak }}\right]}, \quad n \neq n_{\text {peak. }}
$$

5. Use the minimum value of $\mu[n]$ (so that no peak regrowth occurs) as the scaling factor for the whole frame, namely

$$
\mu=\min (\mu[n]) .
$$

6. Scale the clipped signal $\hat{c}_{l}[n]$ by $\mu$ and add it to the original signal to obtain the new transmit signal

$\hat{s}_{l}[n]=s_{l}[n]+\mu \hat{c}_{l}[n], \quad n=[0,1, \cdots,(M-1) N / 2+L-1]$.

7. If $\mu$ is negative, stop the iterative ACE algorithm as any further PAPR reduction attempts will result in peak regrowth.

\subsubsection{Overlapping SGP}

As previously mentioned, the single scaling SGP method presented above scales the clipped signal, which spans the entire frame, by a single scaling factor. This method can be expanded to consider the impact of the individual symbols on the original baseband signal and obtain an optimal scaling vector $\boldsymbol{\mu}$ by which we can scale each individual symbol separately allowing for higher degrees of freedom.
Once again, we obtain the discrete clipped FBMC symbols in isolation, namely $\hat{c}_{l}[n]$. As in Figure 8, each clipped symbol only has an effect on a portion of length $L$ on the original transmit signal.

However, when observing the impulse response of the prototype filter as shown in [5], only the middle half of the impulse response actually has significant values. The rest of the impulse response values are almost negligible. This is also illustrated in Figure 8 where the solid line of $\hat{c}_{l, 4}[n]$ and $\hat{c}_{l, 5}[n]$ represent the significant portion of the clipped signals. Therefore, to calculate a more accurate estimate for $\mu$, we only consider the middle section of length $N$ of the clipped symbols. The peak value of the original transmit signal $s_{l}[n]$ is then calculated between these intervals. This is used to calculate a ratio between the new positive projections and the peak on this interval. In order to calculate the projections $\bar{c}_{l, m}[n]$ of $\hat{c}_{l, m}[n]$ onto the phase angle of the original transmit signal, the dot product can be used. This calculates only the component of $\hat{c}_{l}[n]$ projected on the same phase angle as the original signal. In order to mitigate peak regrowth, the goal is to reduce the magnitude of the peak as much as possible, whilst simultaneously limiting new projections that occur on other samples that may result in peak regrowth. Once again, only positive projections are considered as they indicate peak regrowth.

As can be seen by Figure 8, the significant portion of the clipped signals only overlap on an interval of length $N$ whereas the actual clipped signals overlap over the interval $L=K N$. The scaling of the clipped portions still has an effect on a length $L$ of the original transmit signal.

The overlapping SGP technique can be summarized as follows:

1. Obtain the clipped symbols $\hat{c}_{l, 0}[n], \cdots, \hat{c}_{l, M-1}[n]$.

2. Begin by setting $m=0$.

3. Obtain the projections of $\hat{c}_{l, m}[n]$ on the meaningful intervals of length $N$ as

$$
\bar{c}_{l, m}[k]=\frac{\Re\left\{\hat{c}_{l, m}[k] \cdot s_{l}[n]\right\}}{\left|s_{l}[n]\right|}
$$

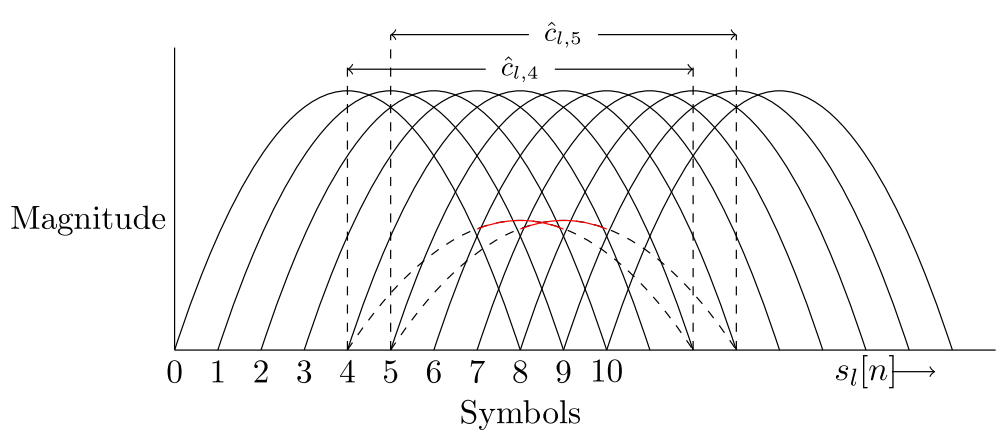

Figure 8 Clipped signals superimposed over original FBMC modulated signal showing significant portion of each symbol. 
with

$$
n=m N / 2+k, \quad 3 N / 2 \leq k \leq 5 N / 2-1 .
$$

4. Find the peak $P_{\boldsymbol{m}}$ on the meaningful interval and its corresponding positions $n_{\text {peak } m}$ as

$$
P_{m}=\max \left|s_{l}[n]\right|
$$

with

$$
\begin{aligned}
m N / 2+3 N / 2 & \leq n \leq m N / 2+5 N / 2, \\
m & =[0,1, \cdots, M-1] .
\end{aligned}
$$

5. Compute the scaling factors for all of the projections by only considering the positive projections, on the meaningful interval calculated in Equation 50, as

$$
\mu_{m}[n]=\frac{P_{m}-\left|s_{l}[n]\right|}{\bar{c}_{l, m}[n]-\bar{c}_{l, m}\left[n_{\text {peak } m}\right] .}
$$

6. Use the minimum value of $\mu_{m}[n]$ as the scaling factor for symbol $m$

$$
\mu_{m}=\min \left(\mu_{m}[n]\right) .
$$

7. Scale the clipped symbol $\hat{c}_{l}[n]$ with its relevant scaling factor $\mu_{m}$ and add it to the original signal $s_{l}[n]$ to obtain the new transmit signal $\hat{s}_{l}[n]$ as

$$
\hat{s}_{l}[n]=s_{l}[n]+\mu_{m} \hat{c}_{q}[n] .
$$

8. Return to step 3 , set $m=m+1$ and update $s_{l}[n]$ with $\hat{s}_{l}[n]$ obtained in step 7 .

9. Once a scaling factor has been obtained for each clipped symbol, a full overlapping SGP iteration has been completed.

10. If the PAPR meets requirements, transmits the new updated $\hat{s}_{l}[n]$, otherwise, repeats the clipping process.

It should be clear from the methodology described above for the overlapping SGP method, scaling values are obtained for each symbol starting at symbol 0 and the transmit signal is updated on a symbol by symbol basis. The updating therefore starts at symbol 0 and propagates through the entire frame until symbol $M-1$ has been updated. This can be considered as a forward progression through the frame. Each consecutive symbol therefore depends on the previous symbol to calculate its respective projections and scaling factor. If a scaling value smaller than zero is obtained for a specific symbol, the scaling factor for that symbol is set to zero as it indicates that scaling will result in peak regrowth. However, the process does not terminate. There may be other symbols that can reduce the PAPR, and so, the scaling factors for other symbols are continued.

The fact that the overlapping SGP proposal depends only on previous symbols implies that a frame-based method of execution is not required. In fact, the overlapping SGP method can be implemented with a delay of only one filter length, namely $L$. It can also be seen at step 6 that the minimum value of $\mu_{m}[n]$ is chosen whereas the proposed optimization methods aim to maximize the $\mu_{m}$ values. The justification for the difference is that the minimization criterion is built into the optimization problem in the form of constraints placed at each sample point, namely Equations 33 to 34 and Equations 38 to 43 .

\section{Comparative performance study}

In this section, we illustrate the performance of the proposed ACE-based PAPR reduction techniques for FBMC techniques using different figures of merit like PAPR, out-of-band distortion and in-band distortion. In addition, we present a study of the power amplifier efficiency improvement allowed with the implementation of the PAPR reduction methods introduced in this work.

In the illustrative simulations, the FBMC system is implemented by using a PHYDYAS-based prototype filter [14] with overlap factor $K=4$. The number of subcarriers is set to $N=64$. It is shown in [14] that when considering near perfect reconstruction orthogonal prototype filters, the choice of prototype filter does not have a significant effect on the PAPR of the FBMC signal. On the other hand, the overlapping factor may in fact have an effect on the PAPR when considering the different techniques (i.e. slightly modified algorithms are required if a different overlap factor is chosen). The choice of $K=4$ is done for accurate comparison to other current methods in the field which employ the same overlap factor. The clipping factor, $\delta$, for all simulated methods, was chosen at $4 \mathrm{~dB}$, and the $\mu$ scaling factor for each symbol in the POCS-based implementation was set to $\mu=1$.

\subsection{PAPR reduction performance}

The CCDF of the PAPR can be used to evaluate the performance of any PAPR reduction technique. This CCDF gives us the information to define an adequate power amplifier operation point, i.e. backoff, with a minimum distortion. The amount of backoff to be applied is a function of the CCDF of the PAPR and the requirements of out-of-band and in-band distortion specified. The PAPR was measured using Equation 11.

Figures 9 and 10 illustrate the results after twice oversampling the PAPR reduction techniques and then applying an oversampling of 8 times to estimate the true analogue nature of a 64 subcarrier, quadrature phase-shift keying (QPSK) system. The final oversampling is required for closer approximation to analogue signals and is achievable in an OFDM or FBMC system by applying a highly up-sampled IFFT block [15]. This allows for better estimation of the peak regrowth in the analogue domain, as the samples in the time domain have some level of correlation.

From the results, it is clear that significant reduction in PAPR performance is achievable with the proposed algorithms. In Figures 9 and 10, PAPR reduction of $3 \mathrm{~dB}$ 


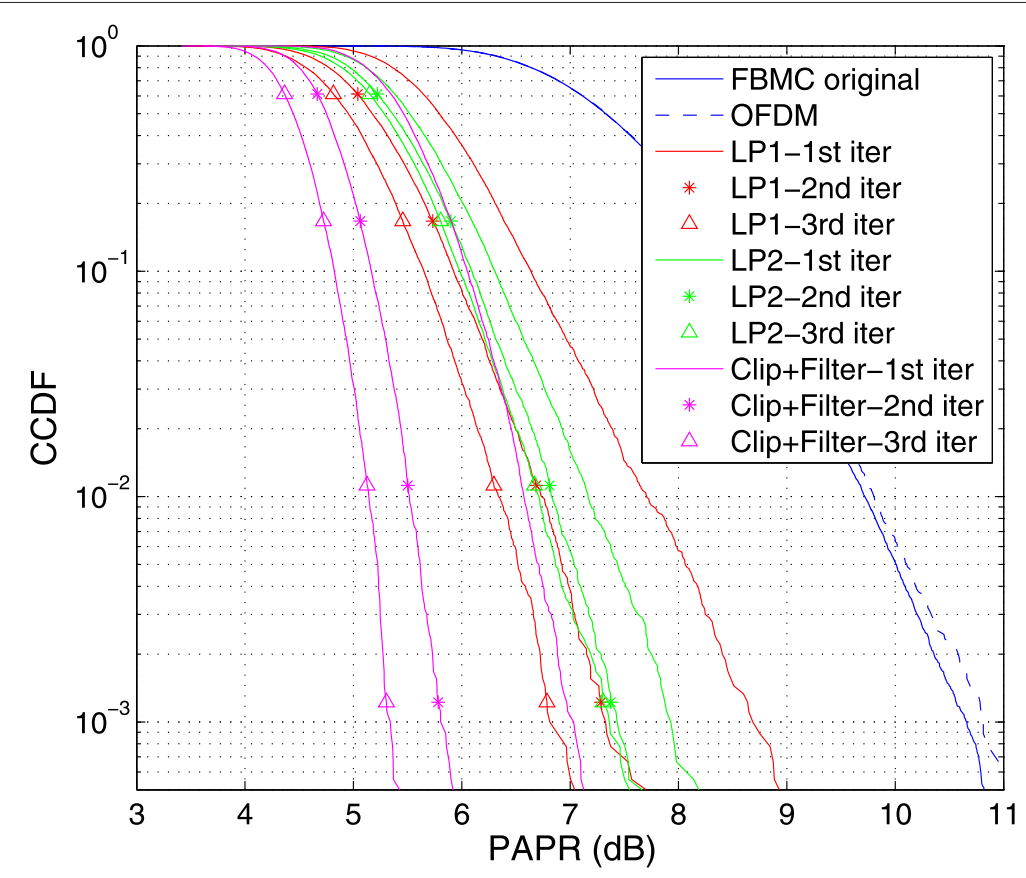

Figure 9 PAPR performance comparison of FBMC, SQPSK modulation employing 64 subcarriers (LP methods).

is achievable in a single iteration of the proposed SGP overlapping and SGP single scaling methods at a clipping probability of $10^{-3}$. This offers significant advantage over a standard POCS approach to FBMC, which only achieves PAPR reduction of approximately $2 \mathrm{~dB}$ after three iterations at the same clipping probability. The LP2 method in
Figures 9 and 10 provides similar PAPR reduction capabilities in a single iteration. The LP1 formulation does not achieve as high reduction upon the first iteration but converges to similar performance upon the third iteration. All the proposed methods obtained PAPR reduction of over $3.5 \mathrm{~dB}$ upon the third iteration, with the SGP overlapping

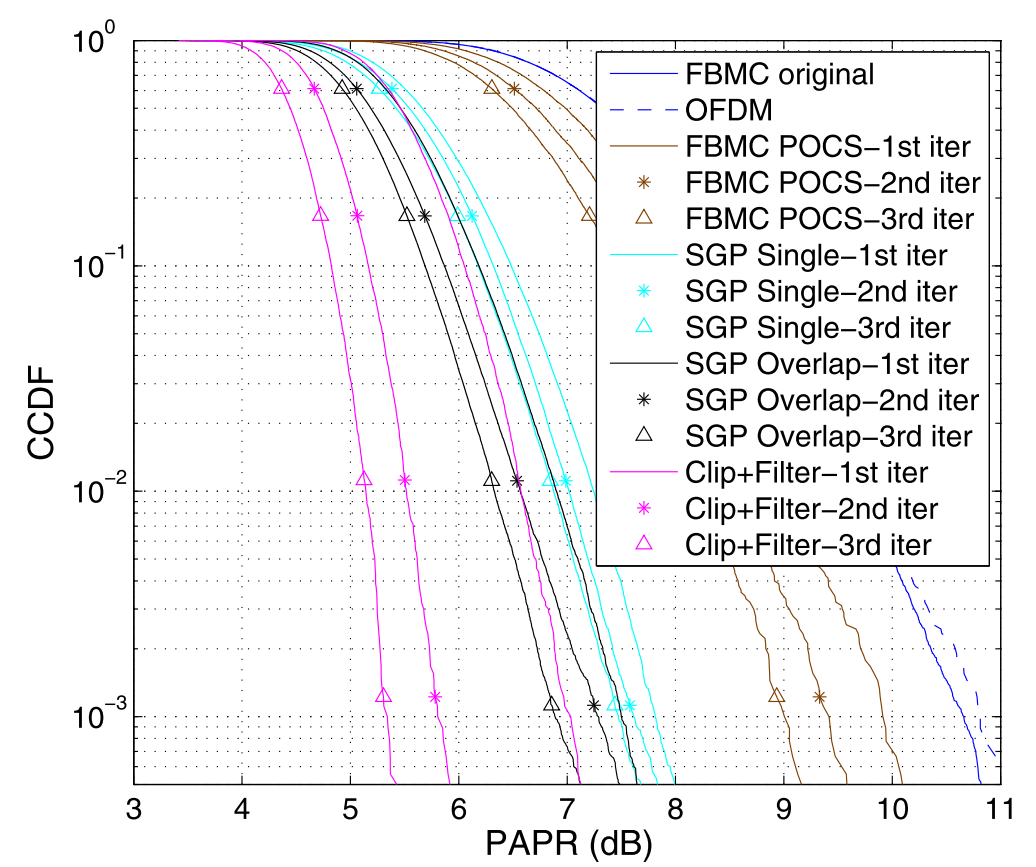

Figure 10 PAPR performance comparison of FBMC, SQPSK modulation employing 64 subcarriers (SGP methods). 
method achieving approximately $3.7 \mathrm{~dB}$ PAPR reduction from the original FBMC envelope at $10^{-3}$ clip probability.

The LP-based proposals provide a benchmark for theoretical upper limit on PAPR reduction capabilities per iteration. It can be seen from Figures 9 and 10 that performance of the SGP overlap method is negligibly close to that of the upper limit per iteration with a much lower level of computation complexity. Therefore, for the rest of the simulations, the LP formulations will be excluded.

The algorithms were expanded to an equivalent 16 QAM modulation-based FBMC to show the effects of higher-order modulation on the PAPR reduction capabilities of the proposed methods. Due to the ACE-based method retention of the minimum Euclidean distance in each constellation, PAPR reduction performance will be less efficient on higher-order modulations. This is due to a reduction in the degrees of freedom in the algorithms as only the outer constellation points can be extended. Figure 11 illustrates the PAPR performance of the proposed methods using 64 subcarriers in oversampled 16 QAM system.

One of the benefits of ACE type PAPR reduction techniques is the scalability as the subcarrier count increases. This normally leads to improved PAPR reduction capabilities as more subcarriers exist to which the ACE method can be applied, effectively providing more handles for controlling the PAPR.

Figures 12 and 13 present the results of 128 subcarrier, oversampled FBMC. It can be seen in Figures 12 and
13 that the PAPR of the original FBMC envelope, when employing $N=128$ subcarriers, has increased by $0.5 \mathrm{~dB}$. The performance of the PAPR reduction techniques, however, remains almost unchanged from the 64 subcarrier simulations presented in Figures 9, 10, 11. This is indicative of an increase in PAPR reduction capabilities as the subcarrier count is increased.

The required amount of power amplifier (PA) backoff is closely related to the PAPR of the multicarrier signal. Large PAPR levels lead to increased backoff and reduced power efficiency.

In order to compare the efficiency of the proposed methods to that found in [8-10], the best case scenarios presented in the literature were compared to the proposed methods. Due to the most current methods in the literature only simulating up to a clip probability of $10^{-3}$ for critical sampled FBMC employing 64 subcarriers, this was decided as the basic metric for comparison. Using the PAPR reduction performance at a clipping probability of $10^{-3}$, it is possible to draw up a baseline for comparison. This comparison can be found in Table 1 with SGP1 the single scaling and SGP2 the overlapping techniques.

\subsection{Out-of-band distortion evaluation}

A matter of critical importance is the spectral leakage of the proposed methods. FBMC has gained much favour due to the very low spectral leakage. However, when a high-power amplifier clips, severe spectral leakage can occur. That is one of the fundamentally important

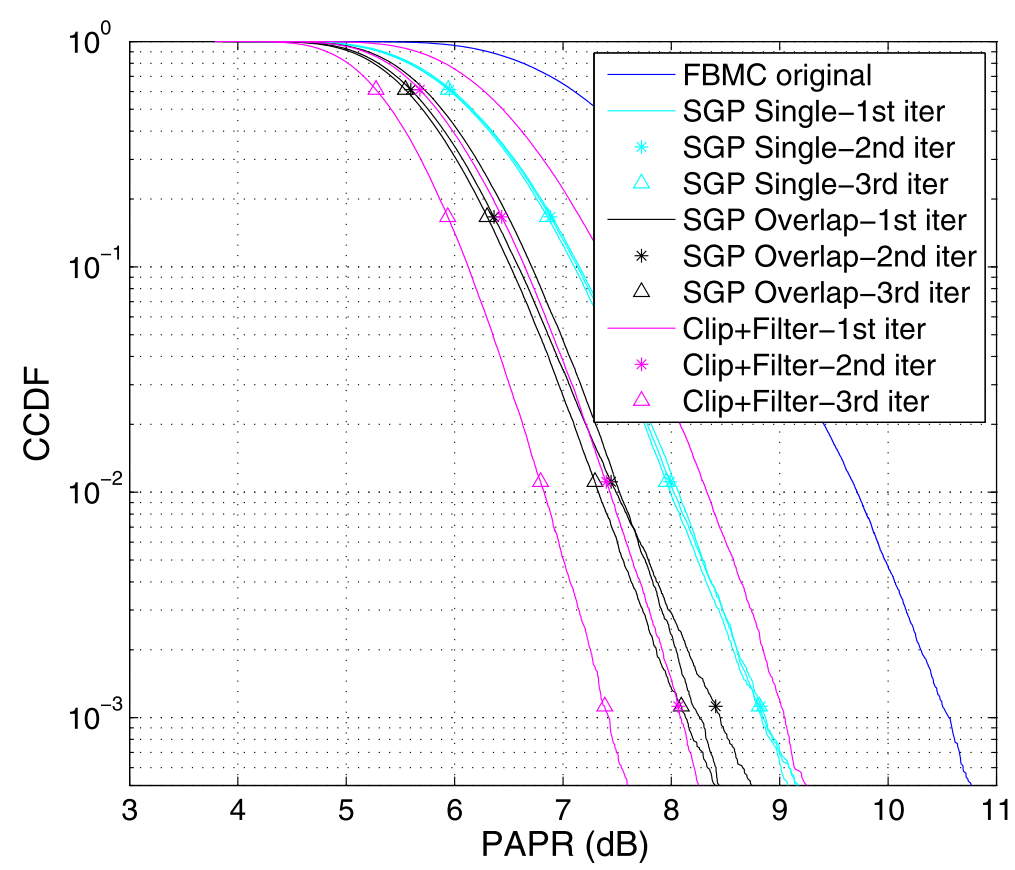

Figure 11 PAPR performance comparison of FBMC, 16 QAM modulation employing 64 subcarriers. 


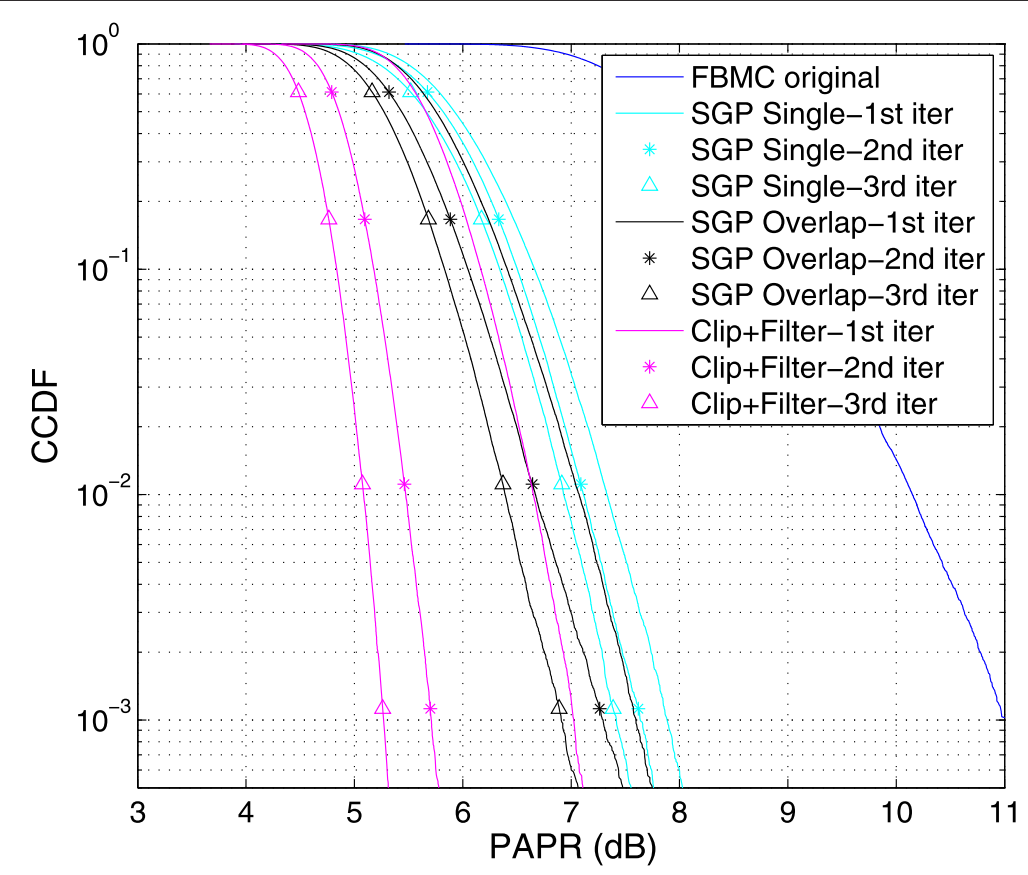

Figure 12 PAPR performance comparison of FBMC, QPSK modulation employing 128 subcarriers.

features of PAPR reduction techniques, i.e. they prevent HPA clipping and therefore reduce unwanted outof-band radiation. In order to accurately simulate and observe the results of the proposed PAPR reduction techniques in terms of power spectral density (PSD), a system was simulated using 64 active subcarriers with twice oversampling prior to 8 times oversampling for estimation to analogue. An ideal class A HPA was simulated with a hard clipping level of $5 \mathrm{~dB}$. This allows regular clipping, and hence, the effects of the out-of-band distortion created by the HPA clipping after PAPR reduction processing can be observed. It should be noted that the PSD only

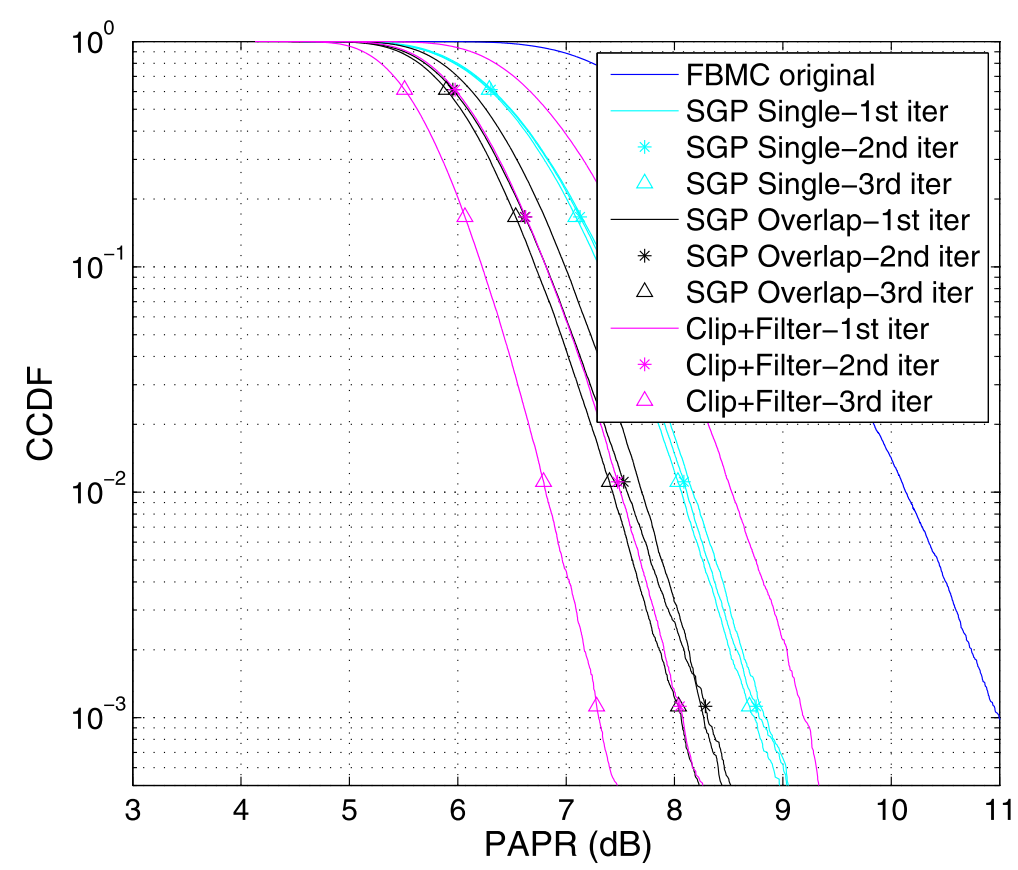

Figure 13 PAPR performance comparison of FBMC, 16 QAM modulation employing 128 subcarriers. 
Table 1 PAPR reduction comparison of 64 subcarrier FBMC modulated systems employing different PAPR reduction techniques

\begin{tabular}{|c|c|c|c|c|c|c|c|c|}
\hline & \multirow[b]{2}{*}{ Original FBMC } & \multicolumn{4}{|c|}{ Proposed PAPR reduction method } & \multicolumn{3}{|c|}{ Current literature } \\
\hline & & SGP1 & SGP2 & LP1 & LP2 & OSLM & SW-TR & Optimized PTS \\
\hline$\overline{\text { PAPR at } 10^{-3}(\mathrm{~dB})}$ & 10.6 & 7.5 & 6.9 & 6.8 & 7.3 & 8.4 & 6.15 & 5.9 \\
\hline
\end{tabular}

gives an indication of the out-of-band leakage and no indication of the in-band distortion caused by HPA clipping. This will be evaluated in terms of BER performance.

The large sidelobe reduction achievable by an unclipped FBMC systems versus unclipped OFDM systems is illustrated in Figures 14 and 15. However, this fundamentally important characteristic, inherent to FBMC, can be severely degraded should clipping occur at the HPA. This can be seen by the FBMC clipped signal in Figures 14 and 15. As in the OFDM scenario, the sidelobe amplitudes are greatly increased, when clipping occurs. This is illustrated when comparing the OFDM ideal/unclipped and FBMC ideal/unclipped plots to the OFDM clipped and FBMC clipped plots. The performance of the proposed techniques is evaluated and it is clear that significant reduction in out-of-band leakage can be achieved, after clipping, when any of the proposed techniques are implemented. However, it is still clear that the rather rudimentary technique of clipping and frequency domain filtering provides the best performance. This is to be expected as the PAPR reduction of the clipping and filtering technique is superior to all other techniques due to no in-band distortion correction.
The ACPR is a figure of merit that can be calculated from Figures 14 and 15 and provides an indication on the spectral leakage into adjacent bands. It is defined as [26]

$$
\mathrm{ACPR}=10 \log _{10}\left(\frac{\int_{f} Y(f) d f}{\int_{f \text { main }} Y(f) d f}\right)
$$

with $Y(f)$ the power spectral distribution at the output of the HPA and $f$ and $f$ main the frequency range of the adjacent channel into which leakage occurs and the main inband channel, respectively. For the ACPR calculations, the main channel was considered between 0 and $2 \mathrm{MHz}$ and adjacent channel was considered between 7 and $9 \mathrm{MHz}$. The results can be found in Table 2 . These results illustrate the benefits of the proposed techniques in cognitive radio environments where ACPR must be minimized in order to mitigate interference between neighbouring bands. This can greatly benefit opportunistic communications where secondary users can now utilize a smaller spectral gap without interfering with the primary users around them.

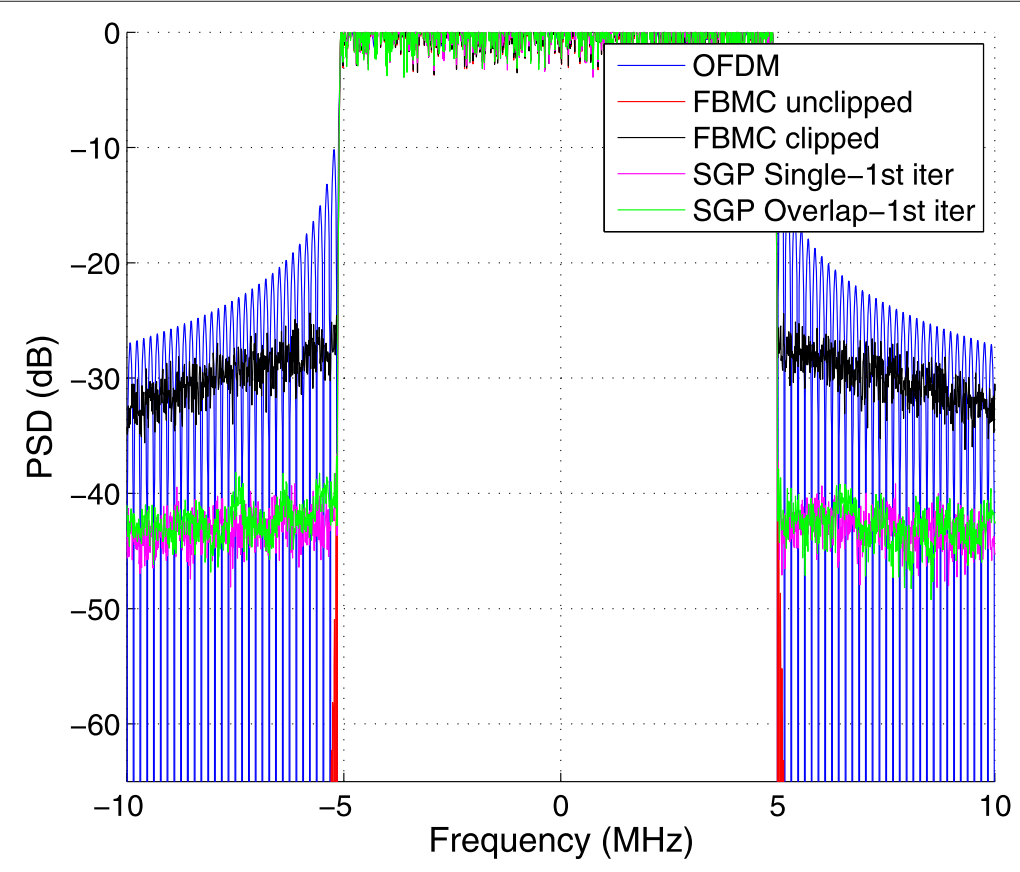

Figure 14 PSD of the proposed PAPR reduction techniques employing 64 active subcarriers. 


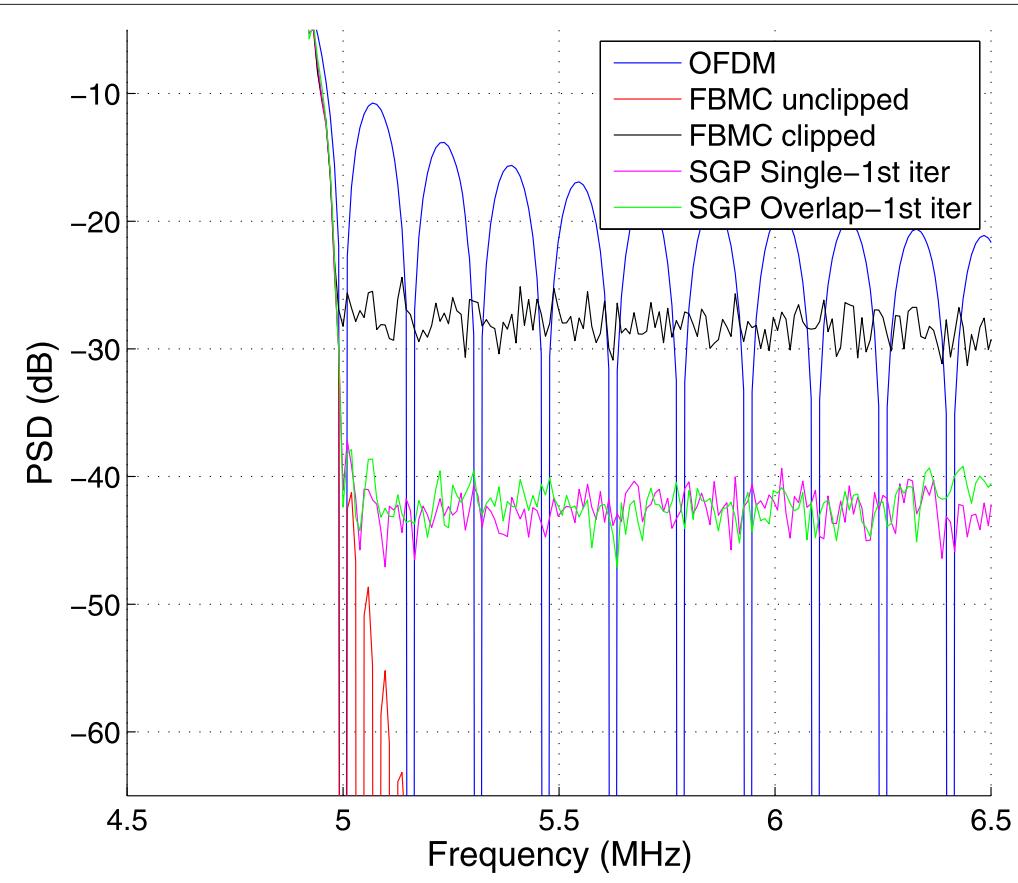

Figure 15 PSD showing sidelobes of the proposed PAPR reduction techniques employing 64 active subcarriers.

\subsection{In-band distortion evaluation}

The final factor for consideration in PAPR reduction techniques, that is often left unattended to, is the matter of BER degradation. The trade-off for all PAPR reduction techniques is a fundamentally important sacrifice in BER performance. This is due to the inherent nature of increase average transmit power, applying constellation distortion or lowering the data rate (which can be modelled as an increase in average bit energy required to transmit the same information).

A figure of merit and often a factor for consideration, when in-band distortion arises, is the error vector magnitude (EVM). This is a measure of the root mean square (RMS) of the absolute distance introduced between the original unmodified constellations and the new distorted constellations. The EVM process can accurately predict BER degradation in distortion techniques as the distortion can be modelled as a random statistical process. However, when considering a constellation extension method such as the ACE technique, the EVM does not provide the same linear relationship between BER degradation and
EVM. This is due to the non-random nature in which the constellations are distorted, as ACE only allows distortion which increase the minimum Euclidean distance. ACE methods do however result in an increase in average transmit power. This factor must be taken into consideration for BER analysis, as the new modified constellation points have larger transmit power than in the unmodified case.

Figure 16 presents the BER results of the proposed techniques, by evaluating the in-band distortion produced by the relevant techniques. These results are produced using a critically sampled, 64 subcarrier QPSK system. It should be noted that Figure 16 does not consider the effects of amplifier clipping distortion and only considers the effects of the distortion inherent in the PAPR reduction techniques prior to amplification. As it is to be expected, there is BER degradation across all techniques. However, it should be noted that, even though the clipping and filtering technique offers superior PAPR reduction capabilities, this comes at the expense of severe in-band distortion. The ACE-based methods mitigate some of

Table 2 ACPR comparison of PAPR reduction techniques with eight times oversampling

\begin{tabular}{|c|c|c|c|c|c|c|c|c|c|c|}
\hline \multirow[b]{2}{*}{ ACPR } & \multicolumn{4}{|c|}{ Original envelope } & \multicolumn{6}{|c|}{ FBMC PAPR reduction method } \\
\hline & $\begin{array}{l}\text { Ideal } \\
\text { FBMC }\end{array}$ & $\begin{array}{l}\text { Clipped } \\
\text { FBMC }\end{array}$ & $\begin{array}{l}\text { Ideal } \\
\text { OFDM }\end{array}$ & $\begin{array}{l}\text { Clipped } \\
\text { OFDM }\end{array}$ & SGP1 & SGP2 & LP1 & LP2 & POCS & Clip + filter \\
\hline First iteration $(\mathrm{dB})$ & -50.6 & -29.84 & -39.49 & -29.18 & -40.13 & -41.19 & -38.84 & -41.44 & -31.38 & -45.19 \\
\hline Third iteration (dB) & -50.6 & -29.84 & -39.49 & -29.18 & -42.56 & -46.61 & -48.73 & -44.97 & -34.13 & -50.6 \\
\hline
\end{tabular}




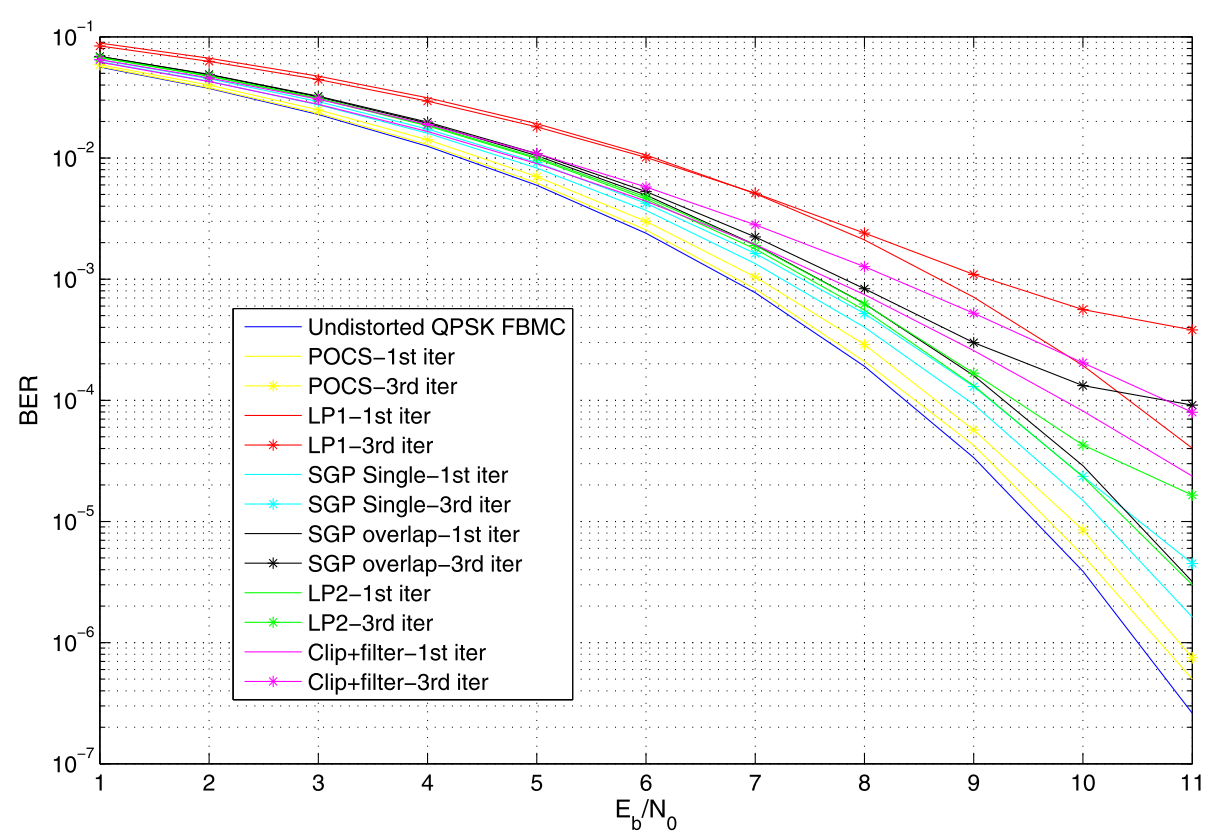

Figure 16 BER results of the proposed FBMC PAPR reduction methods for QPSK modulation.

that distortion by only allowing extensions into allowable regions. It is clear that the single scaling proposal offers the least amount of BER degradation of the proposed methods. This is due to the lower scaling factors inherent of this technique. LP1 seems to offer rather poor BER performance in relation to the other proposals, whilst LP2 provides an acceptable amount of BER degradation even at higher iterations. The first iteration of the overlapping SGP algorithm offers very good BER performance; however, this performance tends to worsen significantly after the first iteration. This can be attributed to the less strict constraints applied to the overlapping SGP proposed method. The tight constraints on LP2 provide excellent BER performance combined with significant PAPR reduction.

The BER performance of all the proposed techniques can be increased by limiting the maximum allowable scaling factor or limiting the maximum allowable constellation extension regions. This allows a hard limit to be placed on the maximum possible increase in average transmit power. This trade-off comes at the expense of slight degradation in PAPR reduction capabilities. However, if the maximum scaling value is chosen smartly, almost negligible sacrifice can be made to PAPR reduction capabilities with significant boosts to BER performance. This is because the large increase in average transmit power comes predominantly from clipped values that are very small, and so, the algorithms tend to favour large scaling values when these situations occur. Due to the very small size in the clipped signals, their peak reducing capabilities are not significant and require large scaling to make a contribution. By limiting the maximum scaling factor, we essentially limit predominantly these points and therefore, almost negligible performance is lost in terms of PAPR reduction capabilities. In fact, a new optimization formulation can be proposed that can set a limit on maximum constellation extension in order to obtain a tight trade-off between PAPR reduction and BER performance degradation caused by constellation distortion.

The BER performance degradation for ACE-based methods is closely related to the average transmit power increase and does not reach an error floor as fast as a hard clipping method. Table 3 illustrates the average power increase across 10,000 frames. The average transmit power increase caused by LP1 is significant at $1 \mathrm{~dB}$ on the first iteration. This closely resembles the BER performance degradation. The rest of the methods follow

Table 3 Average transmit power increase comparison of proposed methods

\begin{tabular}{|c|c|c|c|c|c|c|}
\hline Average power increase & POCS & Clip + filter & SGP single & SGP overlapping & LP1 & LP2 \\
\hline First iteration (dB) & 0.084 & -0.312 & 0.333 & 0.499 & 1.009 & 0.446 \\
\hline Second iteration $(\mathrm{dB})$ & 0.151 & -0.420 & 0.373 & 0.522 & 1.047 & 0.467 \\
\hline
\end{tabular}


similar trends with lower BER degradation corresponding to the lowest power increase of the POCS-based methods.

\subsection{Implementation complexity}

Implementation complexity costs are a serious factor for consideration for any newly proposed technique. Considering the results obtained for the cognitive radio scenario for filtered OFDM and FBMC in [2], both implementation complexities are comparable but high. Adding to this, the iterative characteristic of PAPR reduction techniques may lead to system complexity that is completely infeasible in practice.

For the LP-based problems, each ACE iteration requires the use of the simplex algorithm to solve on optimization problem involving $M$ decision variables and $2((M-1) \times$ $N / 2+L)$ constraints. This can be considered a significantly 'hard' problem to solve, especially when introducing a higher number of subcarriers. The simplex algorithm has in the worst case an exponential time complexity whilst the complexity of a randomized simplex implementation is not known [27]. The per iteration-based complexity of the LP formulated problems therefore far exceeds the proposed SGP methods and most likely renders their practical implementation infeasible. The LP formulations are included as a benchmark for a theoretical upper bound on PAPR performance of the proposed SGP techniques.

The overlapping SGP and single scaling SGP methods involve a dot product computation to calculate the clipped projections for each sample combined with additional signal processing to find the optimal scaling factor per symbol and per frame, respectively. The complexity can be implicitly evaluated by comparing the number of real multiplications present in the proposed techniques. The number of real multiplications of the modulating $C_{\mathrm{SFB}}$ and demodulating $C_{\mathrm{AFB}}$ filter banks can be calculated using [14]

$$
C_{\mathrm{SFB}}=C_{\mathrm{AFB}}=2 \times\left(2 N+N\left(\log _{2}(N)-3\right)+4+2 K N\right) .
$$

The proposed SGP techniques require an additional demodulation and modulation phase prior to transmission, as well as additional signal processing in order to calculate the projections as well as the scaling factors. The projection calculations of Equation 50 can be seen to contribute $4 N$ real multiplications, $N$ real integer divisions and $N$ real square root functions per FBMC symbol. Equation 54 can be calculated at a worst case scenario of $N$ real divisions per FBMC symbol. The net real multiplications required in order to implement the SGP techniques, per SGP iteration, is therefore

$$
C_{\mathrm{SGP}}=6 \times\left(2 N+N\left(\log _{2}(N)-3\right)+4+2 K N\right)+4 N .
$$

Considering $N=64, K=4$,

$$
C_{\mathrm{SFB}}=1,672 \text { real multiplications, }
$$

$$
C_{\mathrm{SGP}}=5,272 \text { real multiplications }
$$

and

$$
\Omega=\frac{C_{\mathrm{SGP}}}{C_{\mathrm{SFB}}}=3.15 \text {. }
$$

Considering $N=128$,

$$
\Omega=\frac{C_{\mathrm{SGP}}}{C_{\mathrm{SFB}}}=3.14 \text {. }
$$

From Equations 62 and 63, we can see that the complexity increase remains constant when the subcarrier count, $N$, increases. This implies that the complexity increase scales linearly with the $N$ or equivalently in big $O$ notation, the additional complexity of the proposed FBMC SGP algorithms can be inferred to be $O(N)$.

\subsection{Discussion}

The PAPR results obtained in Figures 9 and 10 favour a clipping and filtering technique. However, as evident from the BER analysis of Figure 16, the clipping and filtering technique suffers severe BER degradation, introduced by the non-linear in-band distortion inherent of a clipping system. The effects of the distortion tend to diverge the BER curve away from the theoretical QPSK curve due to the error floor being reached faster the more aggressive the clipping. The proposed LP1 technique suffers similar BER degradation after the first iteration, and therefore, the PAPR reduction obtained may not be worth the trade-off. However, of particular interest is the achievable BER in the single scaling and LP2 proposals. Even after the first iteration, the BER is not as significantly degraded as other comparative methods. The overlapping SGP proposal may also offer significant PAPR reduction on the first iteration with minimal BER degradation. However, after the first iteration, any gains associated with PAPR reduction are mitigated by extensive increase in BER degradation. This may be attributed to the less stringent constraints placed on this method, leading to a probability of divergence after the first iteration. This however can be mitigated by limiting the maximum scaling value, thereby ensuring convergence.

The results obtained in Figure 11 for extensions to 16 QAM are less favourable than their QPSK counterparts. This is however to be expected from an ACE-based method as only the outer constellations can be extended. Viability therefore for higher-order modulation schemes is therefore limited. 
As previously mentioned, the trade-off of PAPR reduction comes at the expense of an increase in BER degradation across any PAPR reduction technique [28,29]. In an FBMC scenario, constellation distortion results in BER degradation to the same degree as in OFDM. However, increasing the average transmit power in FBMC also results in an increase in the controlled interference inherent to FBMC systems. This will also contribute to BER degradation. Techniques exist which limit the maximum allowable in-band distortion [30], which if extended to the proposed techniques, may result in significant BER performance gains at negligible PAPR reduction capability loss. In conclusion, an optimal solution still needs to be found between BER performance degradation and PAPR reduction capabilities for FBMC systems such as a decision metric presented in [7].

\section{Conclusions}

The proposed PAPR reduction techniques presented in this paper provide a fast converging ACE alternative to PAPR reduction for FBMC systems. Much like the SGP ACE method for OFDM, an SGP type implementation can be extended to FBMC systems, if the overlapping nature of FBMC is exploited. Significant gains in PAPR reduction capabilities are attained by the proposed methods and compare very favourably with current FBMC PAPR reduction techniques in the literature. The ACPR of unclipped FBMC is maintained after being passed through a high-power amplifier even with input backoff levels as low as $5 \mathrm{~dB}$. BER degradation varies across the proposed methods, and a viable trade-off can be found between PAPR reduction capabilities and BER degradation.

The proposed optimization techniques require framebased processing in order to fully exploit the overlapping nature of FBMC symbols. This would ultimately lead to an increase in system latency in order to account for future symbols and maintain a causal system. This can be partially mitigated by decreasing the frame size over which the optimization occurs. These proposed techniques are therefore ideal candidates for burst transmission. The proposed overlapping SGP however can be implemented in low latency systems. The overlapping SGP technique relies only on past symbols and performs symbol-by-symbol processing. Therefore, a maximum system latency delay of one filter length, $L$, can still be maintained whilst offering significant PAPR reducing capabilities.

Based on the ACPR results obtained, it can be seen that the proposed PAPR reduction methods add further justification to the implementation of FBMC, as a viable successor to OFDM, for future generation networks.

\section{Competing interests}

The authors declare that they have no competing interests.

\section{Author details}

'Department of Electrical, Electronic and Computer Engineering, University of Pretoria, cnr. Lynnwood Road and Roper str., 0002 Pretoria, Republic of South Africa. ${ }^{2}$ CONICET - Department of Electrical and Computer Engineering,

Universidad Nacional del Sur, Av. Alem 1253, 8000 Bahía Blanca, Argentina.

Received: 25 August 2014 Accepted: 21 November 2014

Published: 4 December 2014

\section{References}

1. V Berg, D Noguet, IEEE 1900.7 White space radio, ACLR issues with OFDM for TWWS operation (2011)

2. FP7-ICT-2009-4/248454, QoSMOS, D4.3 Flexible PHY Concepts for White Spaces - Final Report, (2012)

3. S Srinivasan, S Dikmese, M Renfors, in 2012 IEEE 13th International Workshop on Signal Processing Advances in Wireless Communications (SPAWC). Spectrum sensing and spectrum utilization model for OFDM and FBMC based cognitive radios, (2012), pp. 139-143. doi:10.1109/SPAWC.2012.6292874

4. SN Premnath, D Wasden, SK Kasera, B Farhang-Boroujeny, N Patwari, in 2012 Fourth International Conference on Communication Systems and Networks (COMSNETS). Beyond OFDM: Best-effort dynamic spectrum access using filterbank multicarrier, (2012), pp. 1-10

5. M Bellanger, in 2010 IEEE Radio and Wireless Symposium (RWS). Physical layer for future broadband radio systems, (2010), pp. 436-439

6. T Jiang, C Li, C Ni, Effect of PAPR reduction on spectrum and energy efficiencies in OFDM systems with class-A HPA over AWGN channel. IEEE Trans. Broadcast. 59(3), 513-519 (2013). doi:10.1109/TBC.2013.2253814

7. KDhuness, B Maharaj, Analysis of an offset modulation transmission. EURASIP J. Wireless Commun. Netw. 2013(1), 19 (2013). doi:10.1186/1687-1499-2013-19

8. A Skrzypczak, J-P Javaudin, P Siohan, in 2006. VTC 2006-Spring. IEEE 63rd Vehicular Technology Conference, vol. 4. Reduction of the peak-to-average power ratio for the OFDM/OQAM modulation, (2006), pp. 2018-2022. doi:10.1109/NETECS.2006.1683200

9. D Qu, S Lu, T Jiang, Multi-block joint optimization for the peak-to-average power ratio reduction of FBMC-OQAM signals. IEEE Trans. Signal Process. 61(7), 1605-1613 (2013). doi:10.1109/TSP.2013.2239991

10. S Lu, D Qu, Y He, Sliding window tone reservation technique for the peak-to-average power ratio reduction of FBMC-OQAM signals. IEEE Wireless Commun. Lett. 1(4), 268-271 (2012). doi:10.1109/WCL.2012.062512.120360

11. G Cheng, H Li, B Dong, S Li, An improved selective mapping method for PAPR reduction in OFDM/OQAM system. Scientific Res. Publishing Commun. Netw. 5, 53-56 (2013)

12. Z Kollár, L Varga, B Horváth, P Bakki, J Bitó, Evaluation of clipping based iterative PAPR reduction techniques for FBMC systems. The Scientific World Journal. (Hindawi Publishing Corporation, 2014)

13. M Hoch, S Heinrichs, JB Huber, Peak-to-average power ratio and its reduction in wavelet-OFDM. (International OFDM Workshop, Hamburg, Germany, 2011), pp. 56-60

14. Phydyas5.1, PHYDYAS Deliverable D5.1: Prototype Filter and Structure Optimization. (PHYsical layer for DYnamic AccesS and cognitive radio, 2008)

15. BS Krongold, DL Jones, PAR reduction in OFDM via active constellation extension. IEEE Trans. Broadcast. 49(3), 258-268 (2003)

16. B Farhang-Boroujeny, OFDM versus filter bank multicarrier. IEEE Signal Process. Mag. 28(3), 92-112 (2011)

17. Z Kollar, L Varga, K Czimer, in Proceedings of 17 th International OFDM Workshop 2012 (InOWo'12); OFDM 2012. Clipping-based iterative papr-reduction techniques for FBMC, (2012), pp. 1-7

18. P Siohan, C Siclet, N Lacaille, Analysis and design of OFDM/OQAM systems based on filterbank theory. IEEE Trans. Signal Process. 50(5), 1170-1183 (2002)

19. MG Bellanger, G Bonnerot, M Coudreuse, Digital filtering by polyphase network: application to sample-rate alteration and filter banks. IEEE Trans. Acoust. Speech Signal Process. 24(2), 109-114 (1976). doi:10.1109/TASSP.1976.1162788

20. PP Vaidyanathan, Multirate Systems and Filter Banks. (Prentice Hall, Englewood Cliffs, NJ, USA, 1993) 
21. F Schaich, in 2010 European Wireless Conference (EW). Filterbank based multi carrier transmission (FBMC); evolving OFDM: FBMC in the context of WiMAX, (2010), pp. 1051-1058. doi:10.1109/EW.2010.5483518

22. H Ochiai, $\mathrm{H}$ Imai, On the distribution of the peak-to-average power ratio in OFDM signals. IEEE Trans. Commun. 49(2), 282-289 (2001). doi:10.1109/26.905885

23. DS Waldhauser, LG Baltar, JA Nossek, in IEEE Asia Pacific Conference on Circuits and Systems, 2006. APCCAS 2006. Comparison of filter bank based multicarrier systems with OFDM, (2006), pp. 976-979. doi:10.1109/APCCAS.2006.342225

24. SH Han, JH Lee, An overview of peak-to-average power ratio reduction techniques for multicarrier transmission. IEEE Trans. Wireless Commun. 12(2), 56-65 (2005)

25. J Armstrong, in IEEE VTS 53rd Vehicular Technology Conference, 2001. VTC 2001 Spring., vol. 1. New OFDM peak-to-average power reduction scheme, (2001), pp. 756-7601. doi:10.1109/NETECS.2001.944945

26. F Gregorio, J Cousseau, S Werner, T Riihonen, R Wichman, Power amplifier linearization technique with IQ imbalance and crosstalk compensation for broadband MIMO-OFDM transmitters. EURASIP J. Adv. Signal Process. 2011(1), 19 (2011). doi:10.1186/1687-6180-2011-19

27. N Megiddo, On the complexity of linear programming, vol. 12. (Cambridge Univ. Press, 1989). pp. 225-268

28. T Jiang, Y Wu, An Overview: peak-to-average power ratio reduction techniques for OFDM signals. IEEE Trans. Broadcast. 54(2), 257-268 (2008). doi:10.1109/TBC.2008.915770

29. M Niranjan, S Srikanth, in 2011 International Conference on Recent Trends in Information Technology (ICRTIT). Adaptive active constellation extension for PAPR reduction in OFDM systems, (2011), pp. 1186-1189. doi:10.1109/ICRTIT.2011.5972243

30. S-K Deng, M-C Lin, Recursive clipping and filtering with bounded distortion for PAPR reduction. IEEE Trans. Commun. 55(1), 227-230 (2007). doi:10.1109/TCOMM.2006.885102

doi:10.1186/1687-6180-2014-172

Cite this article as: van der Neut et al:: PAPR reduction in FBMC using an ACE-based linear programming optimization. EURASIP Journal on Advances in Signal Processing 2014 2014:172.

\section{Submit your manuscript to a SpringerOpen ${ }^{\circ}$ journal and benefit from:}

- Convenient online submission

- Rigorous peer review

- Immediate publication on acceptance

- Open access: articles freely available online

- High visibility within the field

- Retaining the copyright to your article

Submit your next manuscript at $\boldsymbol{\wedge}$ springeropen.com 